BULLETIN (New Series) OF THE

AMERICAN MATHEMATICAL SOCIETY

Volume 49, Number 3, July 2012, Pages 377-414

S 0273-0979(2012)01372-1

Article electronically published on January 30, 2012

\title{
UNIVERSALITY OF LOCAL SPECTRAL STATISTICS OF RANDOM MATRICES
}

\author{
LÁSZLÓ ERDŐS AND HORNG-TZER YAU
}

\begin{abstract}
The Wigner-Dyson-Gaudin-Mehta conjecture asserts that the local eigenvalue statistics of large random matrices exhibit universal behavior depending only on the symmetry class of the matrix ensemble. For invariant matrix models, the eigenvalue distributions are given by a log-gas with potential $V$ and inverse temperature $\beta=1,2,4$, corresponding to the orthogonal, unitary and symplectic ensembles. For $\beta \notin\{1,2,4\}$, there is no natural random matrix ensemble behind this model, but the statistical physics interpretation of the log-gas is still valid for all $\beta>0$. The universality conjecture for invariant ensembles asserts that the local eigenvalue statistics are independent of $V$. In this article, we review our recent solution to the universality conjecture for both invariant and non-invariant ensembles. We will also demonstrate that the local ergodicity of the Dyson Brownian motion is the intrinsic mechanism behind the universality. Furthermore, we review the solution of Dyson's conjecture on the local relaxation time of the Dyson Brownian motion. Related questions such as delocalization of eigenvectors and local version of Wigner's semicircle law will also be discussed.
\end{abstract}

Perhaps I am now too courageous when I try to guess the distribution of the distances between successive levels (of energies of heavy nuclei). Theoretically, the situation is quite simple if one attacks the problem in a simpleminded fashion. The question is simply what are the distances of the characteristic values of a symmetric matrix with random coefficients.

Eugene Wigner on the Wigner surmise, 1956

\section{INTRODUCTION}

What do the eigenvalues of a typical large matrix look like? Do we expect certain universal patterns of eigenvalue statistics to emerge? Although random matrices appeared already in a concrete statistical application by Wishart in 1928 [77, these natural questions were not raised until the pioneering work [76] of E. Wigner in the 1950s. To make the problem simpler, we restrict ourselves to either real symmetric or complex Hermitian matrices so that the eigenvalues are real. For definiteness, we consider $N \times N$ square matrices $H=H^{(N)}=\left(h_{i j}\right)$ with matrix elements having mean zero and variance $1 / N$, i.e.,

$$
\mathbb{E} h_{i j}=0, \quad \mathbb{E}\left|h_{i j}\right|^{2}=\frac{1}{N} \quad i, j=1,2, \ldots, N .
$$

Received by the editors June 24, 2011, and, in revised form, December 28, 2011.

2010 Mathematics Subject Classification. Primary 15B52, 82B44.

Key words and phrases. Random matrix, local semicircle law, Tracy-Widom distribution, Dyson Brownian motion.

The first author was partially supported by SFB-TR 12 Grant of the German Research Council.

The second author was partially supported by NSF grants DMS-0757425, 0804279 . 
The random variables $h_{i j}, i, j=1, \ldots, N$ are real or complex independent random variables subject to the symmetry constraint $h_{i j}=\bar{h}_{j i}$. These ensembles of random matrices are called Wigner matrices. We will always consider the limit as the matrix size goes to infinity, i.e., $N \rightarrow \infty$.

The first rigorous result about the spectrum of a random matrix of this type is the famous Wigner semicircle law [76 which states that the empirical densities of the eigenvalues, $\lambda_{1}, \lambda_{2}, \ldots, \lambda_{N}$ of large symmetric or Hermitian matrices after proper normalization such as (1.1) are given by

$$
\varrho_{N}(x):=\frac{1}{N} \sum_{j=1}^{N} \delta\left(x-\lambda_{j}\right) \rightarrow \varrho_{s c}(x):=\frac{1}{2 \pi} \sqrt{\left(4-x^{2}\right)_{+}}
$$

in the weak limit as $N \rightarrow \infty$. The limit density is independent of the details of the distribution of $h_{i j}$. The motivation for Wigner was to find a phenomenological model for the energy gap statistics of large atomic nuclei since the energy levels of large quantum systems are impossible to compute from first principles. After several attempts, Wigner was convinced that random matrices were the right models. Besides the semicircle law, he also predicted that the eigenvalue gap distribution in the bulk of the spectrum is given by the Wigner surmise, e.g., in the case of symmetric matrices,

$$
\mathbb{P}\left(\frac{s}{N \varrho} \leq \lambda_{j}-\lambda_{j-1} \leq \frac{s+\mathrm{d} s}{N \varrho}\right) \approx \frac{\pi s}{2} \exp \left(-\frac{\pi}{4} s^{2}\right) \mathrm{d} s,
$$

where $\varrho$ is the local density of eigenvalues (see [51] for an overview).

Wigner's proof of the semicircle law was a moment method via computing $\mathbb{E} \operatorname{Tr} H^{n}$ for each $n$. The Wigner surmise was much more difficult to understand. In the pioneering work by Gaudin [43, the exact gap distributions of random matrices with Gaussian distribution for matrix elements were computed in terms of a Fredholm determinant involving Hermite polynomials. Hermite polynomials were first introduced in the context of random matrices by Mehta and Gaudin 553 earlier. Dyson and Mehta [52, 20, 22, later extended this exact calculation to correlation functions and to other symmetry classes. To keep our presentation simple, we state the corresponding results in terms of the eigenvalue correlation functions for Hermitian $N \times N$ matrices. If $p_{N}\left(\lambda_{1}, \lambda_{2}, \ldots, \lambda_{N}\right)$ denotes the joint probability density of the (unordered) eigenvalues, then the $n$-point correlation functions (marginals) are defined by

$$
p_{N}^{(n)}\left(\lambda_{1}, \lambda_{2}, \ldots, \lambda_{n}\right):=\int_{\mathbb{R}^{N-n}} p_{N}\left(\lambda_{1}, \ldots, \lambda_{n}, \lambda_{n+1}, \ldots, \lambda_{N}\right) \mathrm{d} \lambda_{n+1} \cdots \mathrm{d} \lambda_{N} .
$$

In the Gaussian case, the joint probability density of the eigenvalues can be expressed explicitly as

$$
p_{N}\left(\lambda_{1}, \lambda_{2}, \ldots, \lambda_{N}\right)=\text { const. } \prod_{i<j}\left(\lambda_{i}-\lambda_{j}\right)^{2} \prod_{j=1}^{N} e^{-\frac{1}{2} N \sum_{j=1}^{N} \lambda_{j}^{2}} .
$$

The Vandermonde determinant structure allows one to compute the $k$-point correlation functions in the large $N$ limit via Hermite polynomials that are the orthogonal polynomials with respect to the Gaussian weight function.

The result of Dyson, Gaudin, and Mehta asserts that for any fixed energy $E$ in the bulk of the spectrum, i.e., $|E|<2$, the small scale behavior of $p_{N}^{(n)}$ is given 
explicitly by

$$
\begin{gathered}
\frac{1}{\left[\varrho_{s c}(E)\right]^{n}} p_{N}^{(n)}\left(E+\frac{\alpha_{1}}{N \varrho_{s c}(E)}, E+\frac{\alpha_{2}}{N \varrho_{s c}(E)}, \ldots, E+\frac{\alpha_{n}}{N \varrho_{s c}(E)}\right) \\
\rightarrow \operatorname{det}\left(K\left(\alpha_{i}-\alpha_{j}\right)\right)_{i, j=1}^{n},
\end{gathered}
$$

where $K$ is the celebrated sine kernel

$$
K(x, y)=\frac{\sin \pi(x-y)}{\pi(x-y)} .
$$

Note that the limit in (1.5) is independent of the energy $E$ as long as it is in the bulk of the spectrum. The rescaling by a factor $N^{-1}$ of the correlation functions in (1.5) corresponds to the typical distance between consecutive eigenvalues, and we will refer to the law under such scaling as local statistics. Similar but much more complicated formulas for symmetric matrices were also obtained. It is well known that the eigenvalue gap distribution can be computed from the correlation functions via the inclusion-exclusion principle and thus (1.5) also yields a precise asymptotics for eigenvalue gap distributions. In a striking coincidence, the Wigner surmise, which was based on a $2 \times 2$ matrix computation, agrees with this sophisticated formula with a typical error of only a few percentage points. Note that the correlation functions do not factorize, i.e., the eigenvalues are strongly correlated despite that the matrix elements are independent. Eigenvalues of random matrices thus represent a strongly correlated point process obtained from independent random variables in a natural way.

The central thesis of Wigner is the belief that the eigenvalue gap distributions for large complicated quantum systems are universal in the sense that they depend only on the symmetry class of the physical system but not on other detailed structures. This thesis has never been proved for any truly interacting system, and there is even no heuristically convincing argument for its correctness. Despite this, there is a general belief that the random matrix statistics and Poisson statistics represent two paradigms of energy level statistics for many-body quantum systems: Poisson for independent systems and random matrix for highly correlated systems. In fact, these paradigms extend even to certain one-body systems, such as the quantization of the geodesic flow in a domain, or on a manifold [7, 9], or random Schrödinger operators 66.

In retrospect, Wigner's idea should have received even more attention. For centuries, the primary territory of probability theory was to model uncorrelated or weakly correlated systems via the law of large numbers or the central limit theorem. Random matrix statistics is essentially the first and only general computable pattern for complicated correlated systems, and it is conjectured to be ubiquitous. We only mention here the spectacular result of Montgomery 54 which proves a special case of the conjecture (under the assumption of the Riemann hypothesis) that the distribution of zeros of the Riemann zeta function on the critical line is given by a random matrix statistics.

The simplest class to test Wigner's universality hypothesis upon is the random matrix ensemble itself. All calculations by Dyson, Gaudin, and Mehta are for Gaussian ensembles, i.e., where the matrix elements $h_{i j}$ are real or complex Gaussian random variables. These ensembles are called the Gaussian orthogonal ensemble (GOE) and Gaussian unitary ensemble (GUE). If Wigner's universality hypothesis is correct, then the local eigenvalue statistics should be independent of the law of 
the matrix elements. This is generally referred to as the universality conjecture of random matrices, and we will call it the Wigner-Dyson-Gaudin-Mehta conjecture due to the vision of Wigner and the pioneering work of these authors. It was first formulated in Mehta's treatise on random matrices [51 in 1967 and has remained a key question in the subject ever since. Our goal in this paper is to review the recent progress in this direction and sketch some of the important ideas.

Random matrices have been intensively studied in the last 15-20 years, and we will not be able to present all aspects of this research. We refer the reader to recent comprehensive books [14, 16, 1].

The laws of random matrices can be generally divided into invariant and noninvariant ensembles. The invariant ensembles are characterized by a probability measure of the form $Z^{-1} e^{-N \beta \operatorname{Tr} V(H) / 2} \mathrm{~d} H$, where $N$ is the size of the matrix, $V$ is a real valued potential, and $Z$ is the normalization constant. The parameter $\beta>0$ is determined by the symmetry class of the model and $\mathrm{d} H$ is the Lebesgue measure on matrices in the class. These ensembles are called invariant since the probability law depends only on the trace of a function of the matrix and thus is invariant under changes of coordinates. The matrix elements are in general correlated, and they are independent if only if the model is Gaussian, i.e., $V$ is quadratic.

For invariant ensembles, the probability distribution of the eigenvalues $\lambda=$ $\left(\lambda_{1}, \ldots, \lambda_{N}\right)$ with $\lambda_{1} \leq \cdots \leq \lambda_{N}$ for the measure $e^{-N \beta \operatorname{Tr} V(H) / 2} / Z$ is given by the explicit formula (cf. (1.4))

$$
\begin{aligned}
& \mu_{\beta, V}^{(N)}(\lambda) \mathrm{d} \lambda \sim e^{-\beta N \mathcal{H}(\lambda)} \mathrm{d} \lambda \\
& \text { with Hamiltonian } \mathcal{H}(\lambda):=\sum_{k=1}^{N} \frac{1}{2} V\left(\lambda_{k}\right)-\frac{1}{N} \sum_{1 \leq i<j \leq N} \log \left(\lambda_{j}-\lambda_{i}\right),
\end{aligned}
$$

where the parameter $\beta$ is determined by the symmetry class: $\beta=1$ for symmetric matrices, $\beta=2$ for Hermitian matrices, and $\beta=4$ for self-dual quaternion matrices. The key structural ingredient of this formula, the Vandermonde determinant, is the same as in the Gaussian case (1.4). Thus all previous computations, developed for the Gaussian case, can be carried out for $\beta=1,2,4$ provided that the Gaussian weight function for the orthogonal polynomials is replaced with the function $e^{-\beta V(x) / 2}$. Thus the analysis of correlation functions depends critically on asymptotic properties of the corresponding orthogonal polynomials. In the pioneering work of Dyson, Gaudin, and Mehta, the potential is the quadratic polynomial $V(x)=x^{2} / 2$ and the orthogonal polynomials are the Hermite polynomials whose asymptotic properties are well known.

The extension of this approach to a general potential is a demanding task; important progress was made since the late 1990s by Fokas, Its, and Kitaev 42, Bleher and Its [8], Deift et al. [14, 17, 18, Pastur and Shcherbina [55, 56], and more recently by Lubinsky [50]. These results concern the simpler $\beta=2$ case. For $\beta=1,4$, the universality was established only quite recently for analytic $V$ with additional assumptions [15, 16, 49, 61] using earlier ideas of Widom [75]. The final outcome of these sophisticated analyses is that universality holds for the measure (1.7) in the sense that the short scale behavior of the correlation functions is independent of the potential $V$ (with appropriate assumptions) provided that $\beta$ is one of the classical values, i.e., $\beta \in\{1,2,4\}$, that corresponds to an underlying matrix ensemble. 
Notwithstanding matrix ensembles or orthogonal polynomials, the measure (1.7) is perfectly well defined for any $\beta>0$, and it can be interpreted as the Gibbs measure for a system of particles with a logarithmic interaction (log-gas) at inverse temperature $\beta$. It is therefore a natural question to extend universality to nonclassical $\beta$, but the orthogonal polynomial methods are difficult to apply for this case. For all $\beta>0$, the local statistics for the Gaussian case $V(x)=x^{2} / 2$ can, however, be characterized by the "Brownian carousel" [57, 74] which was derived from a tridiagonal matrix representation [19] of Gaussian random matrices.

Apart from the invariant ensembles, there are many natural non-invariant ensembles; the simplest and most important one being the Wigner ensemble for which the matrix elements are independent subject to a symmetry requirement, e.g., $h_{i j}=\bar{h}_{j i}$ in the Hermitian case. For non-invariant ensembles there is no explicit formula analogous to (1.7) for the joint distribution of the eigenvalues. Hence the methods for the invariant ensembles described above are not applicable. Until very recently, most rigorous results have been on the density of eigenvalues, i.e., the convergence to the the Wigner semicircle law (1.2) was established with certain error estimates; see, e.g., the works by Bai et al. 4] and Guionnet and Zeitouni [44. The universality of the local statistics could only be established for Hermitian Wigner matrices with a substantial Gaussian component by Johansson [46] and Ben Arous and Péché 6]. All previous results on local universality have relied on explicitly computable algebraic formulae. These were provided by orthogonal polynomials in case of the invariant ensembles, and by a modification of the Harish-Chandra/Itzykson/Zuber integral in the case of [46]. Nevertheless, following Wigner's thesis, universality is expected to hold for general Wigner matrices as well.

Having summarized the existing rigorous results that were available until 2008, we set the two main problems we wish to address in this article:

Problem 1. Prove the Wigner-Dyson-Gaudin-Mehta conjecture, i.e., the universality for Wigner matrices with a general distribution for the matrix elements.

Problem 2. Prove the universality of the local statistics for the log-gas (1.7) for all $\beta>0$.

We were able to solve Problem 1 for a very general class of distributions. As for Problem 2, we solved it for the case of real analytic potentials $V$ assuming that the equilibrium measure is supported on a single interval, which, in particular, holds for any convex potential. We now state our results precisely.

Theorem 1.1 (Wigner-Dyson-Gaudin-Mehta conjecture [26, Theorem 7.2]). Suppose that $H=\left(h_{i j}\right)$ is a Hermitian (respectively, symmetric) Wigner matrix. Suppose that for some $\varepsilon>0$

$$
\mathbb{E}\left|\sqrt{N} h_{i j}\right|^{4+\varepsilon} \leq C
$$

for some constant $C$. Let $n \in \mathbb{N}$ and $O: \mathbb{R}^{n} \rightarrow \mathbb{R}$ be compactly supported and continuous. Let $E$ satisfy $-2<E<2$ and let $\xi>0$. Then for any sequence $b_{N}$ 
satisfying $N^{-1+\xi} \leq b_{N} \leq|| E|-2| / 2$, we have

$$
\begin{aligned}
\lim _{N \rightarrow \infty} \int_{E-b_{N}}^{E+b_{N}} \frac{\mathrm{d} E^{\prime}}{2 b_{N}} \int_{\mathbb{R}^{n}} \mathrm{~d} \alpha_{1} \cdots \mathrm{d} \alpha_{n} O\left(\alpha_{1}, \ldots, \alpha_{n}\right) \frac{1}{\varrho_{s c}(E)^{n}} \\
\quad \times\left(p_{N}^{(n)}-p_{\mathrm{G}, N}^{(n)}\right) \times\left(E^{\prime}+\frac{\alpha_{1}}{N \varrho_{s c}(E)}, \ldots, E^{\prime}+\frac{\alpha_{n}}{N \varrho_{s c}(E)}\right) \\
=0 .
\end{aligned}
$$

Here $\varrho_{s c}$ is the semicircle law defined in (1.2), $p_{N}^{(n)}$ is the $n$-point correlation function of the eigenvalue distribution of $H$, and $p_{\mathrm{G}, N}^{(n)}$ is the n-point correlation function of an $N \times N$ GUE (respectively, GOE) matrix.

We remark that the convergence in this theorem is in a weak sense, and it also involves averaging over a small energy interval $E^{\prime} \in\left[E-b_{N}, E+b_{N}\right]$. Stronger types of convergence may also be considered and we will comment on one possible such extension in Section 5. We believe that the issue of convergence types is of a technical nature and it is dwarfed by the challenge to prove universality for the largest possible family of matrix ensembles. The fundamental challenge in random matrix theory remains in answering the question of why random matrix law is ubiquitous for seemingly disparate ensembles and physical systems. We will present a few extensions in this direction in Sections 8 and 10.

In the case of invariant ensembles, it is well known that for $V$ satisfying certain mild conditions the sequence of one-point correlation functions, or densities, associated with $\mu^{(N)}$ has a limit as $N \rightarrow \infty$ and the limiting equilibrium density $\varrho(s)$ can be obtained as the unique minimizer of the functional

$$
I(\nu)=\int_{\mathbb{R}} V(t) \nu(t) \mathrm{d} t-\int_{\mathbb{R}} \int_{\mathbb{R}} \log |t-s| \nu(s) \nu(t) \mathrm{d} t \mathrm{~d} s .
$$

Moreover, for convex $V$ the support of $\varrho$ is a single interval $[A, B]$, and $\varrho$ satisfies the equation

$$
\frac{1}{2} V^{\prime}(t)=\int_{\mathbb{R}} \frac{\varrho(s) \mathrm{d} s}{t-s}
$$

for any $t \in(A, B)$. For the Gaussian case, $V(x)=x^{2} / 2$, the equilibrium density is given by the semicircle law $\varrho=\varrho_{s c}$; see (1.2).

Theorem 1.2 (Bulk universality of $\beta$-ensemble [10, Corollary 2.2]). Assume $V$ is a real analytic function with $\inf _{x \in \mathbb{R}} V^{\prime \prime}(x)>0$. Let $\beta>0$. Consider the $\beta$-ensemble $\mu=\mu_{\beta, V}^{(N)}$ given in (1.7), and let $p_{N}^{(n)}$ denote the $n$-point correlation functions of $\mu$, defined analogously to (1.3). For the Gaussian case, $V(x)=x^{2} / 2$, the correlation functions are denoted by $p_{G, N}^{(n)}$. Let $E \in(A, B)$ lie in the interior of the support of $\varrho$ and similarly let $E^{\prime} \in(-2,2)$ be inside the support of $\varrho_{s c}$. Let $O: \mathbb{R}^{n} \rightarrow \mathbb{R}$ be a smooth, compactly supported function. Then for $b_{N}=N^{-1+\xi}$ with any $0<\xi \leq 1 / 2$, 
we have

$$
\begin{aligned}
\lim _{N \rightarrow \infty} \int \mathrm{d} \alpha_{1} \cdots \mathrm{d} \alpha_{n} O\left(\alpha_{1}, \ldots, \alpha_{n}\right) \\
\times \\
\times \int_{E-b_{N}}^{E+b_{N}} \frac{\mathrm{d} x}{2 b_{N}} \frac{1}{\varrho(E)^{n}} p_{N}^{(n)}\left(x+\frac{\alpha_{1}}{N \varrho(E)}, \ldots, x+\frac{\alpha_{n}}{N \varrho(E)}\right) \\
\left.\quad-\int_{E^{\prime}-b_{N}}^{E^{\prime}+b_{N}} \frac{\mathrm{d} x}{2 b_{N}} \frac{1}{\varrho_{s c}\left(E^{\prime}\right)^{n}} p_{\mathrm{G}, N}^{(n)}\left(x+\frac{\alpha_{1}}{N \varrho_{s c}\left(E^{\prime}\right)}, \ldots, x+\frac{\alpha_{n}}{N \varrho_{s c}\left(E^{\prime}\right)}\right)\right] \\
=0,
\end{aligned}
$$

i.e., the appropriately normalized correlation functions of the measure $\mu_{\beta, V}^{(N)}$ at the level $E$ in the bulk of the limiting density asymptotically coincide with those of the Gaussian case, and they are independent of the value of $E$ in the bulk.

We close this introduction with some short remarks concerning these two theorems. Theorem 1.1 holds for a much larger class of matrix ensembles with independent entries, and we will review some of them in Sections 8 and 10. Although Theorem 1.1 in its current form was proved in [26, the key ideas have been developed through several important steps in [27, 33, 36, 37, 38. In particular, the WignerDyson-Gaudin-Mehta (WDGM) conjecture for Hermitian matrices was first solved in 27] in a joint work with the current authors and Péché, Ramírez, and Schlein. This result holds whenever the distributions of matrix elements are smooth. The smoothness requirement was partially removed in 67 and completely removed in a joint paper with Ramírez, Schlein, Tao, and Vu in [28]. The WDGM conjecture for symmetric matrices was resolved in 33 . In this paper, a novel idea based on Dyson Brownian motion was discovered. The most difficult case, the real symmetric Bernoulli matrices, was solved in [37] where a "Fluctuation Averaging Lemma" (Lemma 3.4 of the current paper) exploiting cancellation of matrix elements of the Green function was first introduced. We will give a more detailed historical review in Section 11 .

The proof of Theorem 1.1 consists of the following three steps, discussed in Sections 3, 2, and 4, respectively. Our three-step strategy was first introduced in 27.

Step 1. Local semicircle law and delocalization of eigenvectors. It states that the density of eigenvalues is given by the semicircle law not only as a weak limit on macroscopic scales (1.2), but also in a strong sense and down to short scales containing only $N^{\varepsilon}$ eigenvalues for all $\varepsilon>0$. This will imply the rigidity of eigenvalues, i.e., that the eigenvalues are near their classical location in the sense to be made clear in Section 2. We also obtain precise estimates on the matrix elements of the Green function which in particular imply complete delocalization of eigenvectors.

Step 2. Universality for Gaussian divisible ensembles. The Gaussian divisible ensembles are matrices of the form $H_{t}=e^{-t / 2} H_{0}+\sqrt{1-e^{-t}} U$, where $H_{0}$ is a Wigner matrix and $U$ is an independent GUE matrix. The parametrization of $H_{t}$ reflects that it is most conveniently obtained by an Ornstein-Uhlenbeck process. There are two methods, and both methods imply the bulk universality of $H_{t}$ for $t=N^{-\tau}$ for 
the entire range of $0<\tau<1$ with different estimates:

2a. Proposition 3.1 of [27] which uses an extension of Johansson's formula [46];

2b. Local ergodicity of the Dyson Brownian motion (DBM).

The approach in 2a yields a slightly stronger estimate than the approach in $2 \mathrm{~b}$, but it works only in the Hermitian case. In this review, we will focus on the Dyson Brownian approach.

Step 3. Approximation by Gaussian divisible ensembles. It is a simple density argument in the space of matrix ensembles which shows that for any probability distribution of the matrix elements there exists a Gaussian divisible distribution with a small Gaussian component, as in Step 2, such that the two associated Wigner ensembles have asymptotically identical local eigenvalue statistics. The first implementation of this approximation scheme was via a reverse heat flow argument [27; it was later replaced by the Green function comparison theorem [36].

The proof of Theorem 1.2 consists of the following two steps that will be presented in Sections 6 and 7

Step 1. Rigidity of eigenvalues. This establishes that the location of the eigenvalues are not too far from their classical locations determined by the equilibrium density $\varrho(s)$.

Step 2. Uniqueness of local Gibbs measures with logarithmic interactions. With the precision of eigenvalue location estimates from Step 1 as an input, the eigenvalue spacing distributions are shown to be given by the corresponding Gaussian ones. (We will take the uniqueness of the spacing distributions as our definition of the uniqueness of Gibbs state.)

There are several similarities and differences between these two methods. Both start with rigidity estimates on eigenvalues and then establish that the local spacing distributions are the same as in the Gaussian cases. The Gaussian divisible ensembles, which play a key role in our theory for noninvariant ensembles, are completely absent for invariant ensembles. The key connection between the two methods, however, is the usage of DBM (or its analogue) in the Steps 2. In Section 2 we will first present this idea.

The method for the proof of Theorem 1.1 is extremely general. As of this writing, it has been applied to the generalized Wigner ensembles, the sample covariance ensembles and the Erdős-Rényi matrices for certain range of the sparseness parameter. It can also be extended to the edges of the spectrum, and it yields edge universality under more general conditions than were previously known. This will be reviewed in Section 9. Extensions to generalized Wigner matrices and ErdősRényi matrices will also be discussed in Sections 8 and 10, As the proof of Theorem 1.2 was just completed, we do not know how far this method can reach; currently we can generalize the result to the nonconvex case under the assumption that the equilibrium measure $\rho$ is supported on a single interval [11. The theory we have developed to prove Theorems 1.1 and 1.2 is purely analytic, and we believe that it unveils the genuine mechanism of the Wigner-Dyson-Gaudin-Mehta universality. Finally, a short summary concerning the recent history of universality is given in Section 11 


\section{Dyson Brownian motion and the Local RELAXation Flow}

2.1. Concept and results. The Dyson Brownian motion (DBM) describes the evolution of the eigenvalues of a Wigner matrix as an interacting point process if each matrix element $h_{i j}$ evolves according to independent (up to symmetry restriction) Brownian motions. We will slightly alter this definition by generating the dynamics of the matrix elements by an Ornstein-Uhlenbeck (OU) process which leaves the standard Gaussian distribution invariant. In the Hermitian case, the OU process for the rescaled matrix elements $v_{i j}:=N^{1 / 2} h_{i j}$ is given by the stochastic differential equation

$$
\mathrm{d} v_{i j}=\mathrm{d} \beta_{i j}-\frac{1}{2} v_{i j} \mathrm{~d} t, \quad i, j=1,2, \ldots, N,
$$

where $\beta_{i j}, i<j$, are independent complex Brownian motions with variance one and $\beta_{i i}$ are real Brownian motions of the same variance. Denote the distribution of the eigenvalues $\lambda=\left(\lambda_{1}, \lambda_{2}, \ldots, \lambda_{N}\right)$ of $H_{t}$ at time $t$ by $f_{t}(\lambda) \mu_{G}(\mathrm{~d} \lambda)$, where $\mu_{G}$ is given by (1.7) with the potential $V(x)=x^{2} / 2$.

Then $f_{t}=f_{t, N}$ satisfies ([21])

$$
\partial_{t} f_{t}=\mathscr{L} f_{t}
$$

where

$$
\mathscr{L}=\mathscr{L}_{N}:=\sum_{i=1}^{N} \frac{1}{2 N} \partial_{i}^{2}+\sum_{i=1}^{N}\left(-\frac{\beta}{4} \lambda_{i}+\frac{\beta}{2 N} \sum_{j \neq i} \frac{1}{\lambda_{i}-\lambda_{j}}\right) \partial_{i}, \quad \partial_{i}=\frac{\partial}{\partial \lambda_{i}} .
$$

The parameter $\beta$ is chosen as follows: $\beta=2$ for complex Hermitian matrices and $\beta=1$ for symmetric real matrices. Our formulation of the problem has already taken into account Dyson's observation that the invariant measure for this dynamics is $\mu_{G}$. A natural question regarding the DBM is how fast the dynamics reaches equilibrium. Dyson had already posed this question in 1962:

Dyson's conjecture ([21]). The global equilibrium of DBM is reached in time of order one and the local equilibrium (in the bulk) is reached in time of order $1 / N$. Dyson further remarked,

The picture of the gas coming into equilibrium in two well-separated stages, with microscopic and macroscopic time scales, is suggested with the help of physical intuition. A rigorous proof that this picture is accurate would require a much deeper mathematical analysis.

We will prove that Dyson's conjecture is correct if the initial data of the flow is a Wigner ensemble, which was Dyson's original interest. Our result in fact is valid for DBM with much more general initial data that we now survey. Briefly, it will turn out that the global equilibrium is indeed reached within a time of order one, but local equilibrium is achieved much faster if an a priori estimate on the location of the eigenvalues (also called points) is satisfied. To formulate this estimate, let $\gamma_{j}=\gamma_{j, N}$ denote the location of the $j$-th point under the semicircle law, i.e., $\gamma_{j}$ is defined by

$$
N \int_{-\infty}^{\gamma_{j}} \varrho_{s c}(x) \mathrm{d} x=j, \quad 1 \leq j \leq N .
$$

We will call $\gamma_{j}$ the classical location of the $j$-th point. 
A priori estimate. There exists an $\mathfrak{a}>0$ such that

$$
Q=Q_{\mathfrak{a}}:=\sup _{t \geq N^{-2 \mathfrak{a}}} \frac{1}{N} \int \sum_{j=1}^{N}\left(\lambda_{j}-\gamma_{j}\right)^{2} f_{t}(\lambda) \mu_{G}(\mathrm{~d} \lambda) \leq C N^{-1-2 \mathfrak{a}}
$$

with a constant $C$ uniformly in $N$. (This a priori estimate was referred to as Assumption III in 33, 34.)

The main result on the local ergodicity of Dyson Brownian motion states that if the a priori estimate (2.5) is satisfied, then the local correlation functions of the measure $f_{t} \mu_{G}$ are the same as the corresponding ones for the Gaussian measure, $\mu_{G}=f_{\infty} \mu_{G}$, provided that $t$ is larger than $N^{-2 \mathfrak{a}}$. The $n$-point correlation functions of the probability measure $f_{t} \mathrm{~d} \mu_{G}$ are defined, similarly to (1.3), by

$$
p_{t, N}^{(n)}\left(x_{1}, x_{2}, \ldots, x_{n}\right)=\int_{\mathbb{R}^{N-n}} f_{t}(\mathbf{x}) \mu_{G}(\mathbf{x}) \mathrm{d} x_{n+1} \cdots \mathrm{d} x_{N}, \quad \mathbf{x}=\left(x_{1}, x_{2}, \ldots, x_{N}\right) .
$$

Due to the convention that one can view the locations of eigenvalues as the coordinates of particles, we have used $\mathbf{x}$, instead of $\boldsymbol{\lambda}$, in the last equation. From now on, we will use both conventions depending on which viewpoint we wish to emphasize. Notice that the probability distribution of the eigenvalues at the time $t, f_{t} \mu_{G}$, is the same as that of the Gaussian divisible matrix

$$
H_{t}=e^{-t / 2} H_{0}+\left(1-e^{-t}\right)^{1 / 2} U,
$$

where $H_{0}$ is the initial Wigner matrix and $U$ is an independent standard GUE (or GOE) matrix. This establishes the universality of the Gaussian divisible ensembles. The precise statement is the following theorem.

Theorem 2.1 (34, Theorem 2.1]). Suppose that the a priori estimate (2.5) holds for the solution $f_{t}$ of the forward equation (2.2) with some exponent $\mathfrak{a}>0$. Let $E \in(-2,2)$ and $b>0$ be such that $[E-b, E+b] \subset(-2,2)$. Then for any $s>0$, for any integer $n \geq 1$, and for any compactly supported continuous test function $O: \mathbb{R}^{n} \rightarrow \mathbb{R}$, we have

$$
\begin{aligned}
\lim _{N \rightarrow \infty} \sup _{t \geq N^{-2 \mathfrak{a}+s}} & \int_{E-b}^{E+b} \frac{\mathrm{d} E^{\prime}}{2 b} \int_{\mathbb{R}^{n}} \mathrm{~d} \alpha_{1} \cdots \mathrm{d} \alpha_{n} O\left(\alpha_{1}, \ldots, \alpha_{n}\right) \frac{1}{\varrho_{s c}(E)^{n}} \\
& \times\left(p_{t, N}^{(n)}-p_{G, N}^{(n)}\right)\left(E^{\prime}+\frac{\alpha_{1}}{N \varrho_{s c}(E)}, \ldots, E^{\prime}+\frac{\alpha_{n}}{N \varrho_{s c}(E)}\right)=0 .
\end{aligned}
$$

We can choose $b=b_{N}$ depending on $N$. In 34 explicit bounds on the speed of convergence and the optimal range of $b$ were also established. In particular, thanks to the optimal rigidity estimate [38, i.e., (2.5) with $\mathfrak{a}=1 / 2$, the range of the energy averaging in (2.8) was improved to $b_{N} \geq N^{-1+\xi}, \xi>0$, but only for $t \geq N^{-\xi / 8}$ (Theorem 2.3 of 38 ).

Theorem 2.1 is a consequence of the following theorem, which identifies the gap distribution of the eigenvalues.

Theorem 2.2 (Universality of the Dyson Brownian motion for short time [34, Theorem 4.1]). Suppose $\beta \geq 1$, and let $G: \mathbb{R} \rightarrow \mathbb{R}$ be a smooth function with compact 
support. Then for any sufficiently small $\varepsilon>0$, independent of $N$, there exist constants $C, c>0$, depending only on $\varepsilon$ and $G$ such that for any $J \subset\{1,2, \ldots, N-1\}$ we have

$$
\begin{aligned}
& \left|\int \frac{1}{|J|} \sum_{i \in J} G\left(N\left(x_{i}-x_{i+1}\right)\right) f_{t} \mathrm{~d} \mu_{G}-\int \frac{1}{|J|} \sum_{i \in J} G\left(N\left(x_{i}-x_{i+1}\right)\right) \mathrm{d} \mu_{G}\right| \\
& \quad \leq C N^{\varepsilon} \sqrt{\frac{N^{2} Q}{|J| t}}+C e^{-c N^{\varepsilon}} .
\end{aligned}
$$

In particular, if the a priori estimate (2.5) holds with some $\mathfrak{a}>0$ and $|J|$ is of order $N$, then for any $t>N^{-2 \mathfrak{a}+3 \varepsilon}$ the right-hand side converges to zero as $N \rightarrow \infty$, i.e., the gap distributions for $f_{t} \mathrm{~d} \mu_{G}$ and $\mathrm{d} \mu_{G}$ coincide.

The test functions can be generalized to

$$
G\left(N\left(x_{i}-x_{i+1}\right), N\left(x_{i+1}-x_{i+2}\right), \ldots, N\left(x_{i+n-1}-x_{i+n}\right)\right)
$$

for any $n$ fixed, which is needed to identify higher order correlation functions. In applications, $J$ is chosen to be the indices of the eigenvalues in the interval $[E-b, E+b]$ and thus $|J| \sim N b$. This identifies the gap distributions of eigenvalues completely, and thus also identifies the correlation functions and concludes Theorem 2.1. Note that the input of this theorem, the a priori estimate (2.5), identifies the location of the eigenvalues only on a scale $N^{-1 / 2-\mathfrak{a}}$, which is much weaker than the $1 / N$ precision for the eigenvalue differences in (2.9).

By the rigidity estimates (see Corollary 3.2 below), the a priori estimate (2.5) holds for any $\mathfrak{a}<1 / 2$ if the initial data of the DBM is a Wigner ensemble. Therefore, Theorem 2.2 holds for any $t \geq N^{-1+\varepsilon}$ for any $\varepsilon>0$, and this establishes Dyson's conjecture.

2.2. Main ideas behind the proof of Theorem 2.2, The key method is to analyze the relaxation to equilibrium of the dynamics (2.2). This approach was first introduced in Section 5.1 of [33] the presentation here follows [34].

We start with a short review of the logarithmic Sobolev inequality for a general measure. Let the probability measure $\mu$ on $\mathbb{R}^{N}$ be given by a general Hamiltonian $\mathcal{H}$,

$$
\mathrm{d} \mu(\mathbf{x})=\frac{e^{-N \mathcal{H}(\mathbf{x})}}{Z} \mathrm{~d} \mathbf{x},
$$

and let $\mathscr{L}$ be the generator, symmetric with respect to the measure d $\mu$, defined by the associated Dirichlet form

$$
D(f)=D_{\mu}(f)=-\int f \mathscr{L} f \mathrm{~d} \mu:=\frac{1}{2 N} \sum_{j} \int\left(\partial_{j} f\right)^{2} \mathrm{~d} \mu, \quad \partial_{j}=\partial_{x_{j}} .
$$

Recall the relative entropy of two probability measures:

$$
S(\nu \mid \mu):=\int \frac{\mathrm{d} \nu}{\mathrm{d} \mu} \log \left(\frac{\mathrm{d} \nu}{\mathrm{d} \mu}\right) \mathrm{d} \mu .
$$

If $\mathrm{d} \nu=f \mathrm{~d} \mu$, then we will sometimes use the notation $S_{\mu}(f):=S(f \mu \mid \mu)$. The entropy can be used to control the total variation norm via the well known inequality

$$
\int|f-1| \mathrm{d} \mu \leq \sqrt{2 S_{\mu}(f)}
$$


Let $f_{t}$ be the solution to the evolution equation

$$
\partial_{t} f_{t}=\mathscr{L} f_{t}, \quad t>0
$$

with a given initial condition $f_{0}$. The evolution of the entropy $S_{\mu}\left(f_{t}\right)=S\left(f_{t} \mu \mid \mu\right)$ satisfies

$$
\partial_{t} S_{\mu}\left(f_{t}\right)=-4 D_{\mu}\left(\sqrt{f_{t}}\right) .
$$

By Bakry and Émery [5], the evolution of the Dirichlet form satisfies the inequality

$$
\partial_{t} D_{\mu}\left(\sqrt{f_{t}}\right) \leq-\frac{1}{2 N} \int\left(\nabla \sqrt{f}_{t}\right)\left(\nabla^{2} \mathcal{H}\right) \nabla \sqrt{f} \mathrm{~d} \mu .
$$

If the Hamiltonian is convex, i.e.,

$$
\nabla^{2} \mathcal{H}(\mathbf{x})=\operatorname{Hess} \mathcal{H}(\mathbf{x}) \geq \vartheta \quad \text { for all } \mathbf{x} \in \mathbb{R}^{N}
$$

with some constant $\vartheta>0$, then we have

$$
\partial_{t} D_{\mu}\left(\sqrt{f_{t}}\right) \leq-\vartheta D_{\mu}\left(\sqrt{f_{t}}\right) .
$$

Integrating (2.15) and (2.18) back from infinity to 0, we obtain the logarithmic Sobolev inequality (LSI)

$$
S_{\mu}(f) \leq \frac{4}{\vartheta} D_{\mu}(\sqrt{f}), \quad f=f_{0}
$$

and the exponential relaxation of the entropy and Dirichlet form on time scale $t \sim 1 / \vartheta$

$$
S_{\mu}\left(f_{t}\right) \leq e^{-t \vartheta} S_{\mu}\left(f_{0}\right), \quad D_{\mu}\left(\sqrt{f_{t}}\right) \leq e^{-t \vartheta} D_{\mu}\left(\sqrt{f_{0}}\right) .
$$

As a consequence of the LSI, we also have the concentration inequality for any $k$ and $a>0$

$$
\int \mathbf{1}\left(\left|x_{k}-\mathbb{E}_{\mu}\left(x_{k}\right)\right|>a\right) \mathrm{d} \mu \leq 2 e^{-\vartheta N a^{2} / 2} .
$$

We will not use this inequality in this section, but it will become important in Section 6 .

Returning to the classical ensembles, we assume from now on that $\mathcal{H}$ is given by (1.7) with $V(x)=x^{2} / 2$ and the equilibrium measure is the Gaussian one, $\mu=\mu_{G}$. We then have the convexity inequality

$$
\left\langle\mathbf{v}, \nabla^{2} \mathcal{H}(\mathbf{x}) \mathbf{v}\right\rangle \geq \frac{1}{2}\|\mathbf{v}\|^{2}+\frac{1}{N} \sum_{i<j} \frac{\left(v_{i}-v_{j}\right)^{2}}{\left(x_{i}-x_{j}\right)^{2}} \geq \frac{1}{2}\|\mathbf{v}\|^{2}, \quad \mathbf{v} \in \mathbb{R}^{N} .
$$

This guarantees that $\mu$ satisfies the LSI with $\vartheta=1 / 2$ and the relaxation time to equilibrium is of order one.

The key idea is that the relaxation time is in fact much shorter than order one for local observables that depend only on the eigenvalue differences. Equation (2.22) shows that the relaxation in the direction $v_{i}-v_{j}$ is much faster than order one provided that $x_{i}-x_{j}$ are close. However, this effect is hard to exploit directly due to that all modes of different wavelengths are coupled. Our idea is to add an auxiliary strongly convex potential $W(\mathbf{x})$ to the Hamiltonian to "speed up" the convergence to local equilibrium. On the other hand, we will also show that the cost of this speeding up can be effectively controlled if the a priori estimate (2.5) holds. 
The auxiliary potential $W(\mathbf{x})$ is defined by

$$
W(\mathbf{x}):=\sum_{j=1}^{N} W_{j}\left(x_{j}\right), \quad W_{j}(x):=\frac{1}{2 \tau}\left(x_{j}-\gamma_{j}\right)^{2},
$$

i.e., it is a quadratic confinement on scale $\sqrt{\tau}$ for each eigenvalue near its classical location, where the parameter $\tau>0$ will be chosen later. The total Hamiltonian is given by

$$
\widetilde{\mathcal{H}}:=\mathcal{H}+W,
$$

where $\mathcal{H}$ is the Gaussian Hamiltonian given by (1.7). The measure with Hamiltonian $\widetilde{\mathcal{H}}$,

$$
\mathrm{d} \omega:=\omega(\mathbf{x}) \mathrm{d} \mathbf{x}, \quad \omega:=e^{-N \widetilde{\mathcal{H}}} / \widetilde{Z},
$$

will be called the local relaxation measure. This measure was named the pseudoequilibrium measure in our previous papers.

The local relaxation flow is defined to be the flow with the generator characterized by the natural Dirichlet form w.r.t. $\omega$, explicitly, $\widetilde{\mathscr{L}}$ :

$$
\widetilde{\mathscr{L}}=\mathscr{L}-\sum_{j} b_{j} \partial_{j}, \quad b_{j}=W_{j}^{\prime}\left(x_{j}\right)=\frac{x_{j}-\gamma_{j}}{\tau} .
$$

We will typically choose $\tau \ll 1$ so that the additional term $W$ substantially increases the lower bound (2.17) on the Hessian, hence speeding up the dynamics so that the relaxation time is at most $\tau$.

The idea of adding an artificial potential $W$ to speed up the convergence appears to be unnatural here. The current formulation is a streamlined version of a much more complicated approach that appeared in [33] and which took ideas from the earlier work [29]. Roughly speaking, in hydrodynamical limit, the short wavelength modes always have shorter relaxation times than the long wavelength modes. A direct implementation of this idea is extremely complicated due to the logarithmic interaction that couples short and long wavelength modes. Adding a strongly convex auxiliary potential $W(\mathbf{x})$ shortens the relaxation time of the long wavelength modes, but it does not affect the short modes, i.e., the local statistics, which are our main interest. The analysis of the new system is much simpler since now the relaxation is faster, uniform for all modes. Finally, we need to compare the local statistics of the original system with those of the modified one. It turns out that the difference is governed by $(\nabla W)^{2}$, which can be directly controlled by the a priori estimate (2.5).

Our method for enhancing the convexity of $\mathcal{H}$ is reminiscent of a standard convexification idea concerning metastable states. To explain the similarity, consider a particle near one of the local minima of a double well potential separated by a local maximum, or energy barrier. Although the potential is not convex globally, one may still study a reference problem defined by convexifying the potential along with the well in which the particle initially resides. Before the particle reaches the energy barrier, there is no difference between these two problems. Thus questions concerning time scales shorter than the typical escape time can be conveniently answered by considering the convexified problem; in particular the escape time in the metastability problem itself can be estimated by using convex analysis. Our DBM problem is already convex, but not sufficiently convex. The modification by adding $W$ enhances convexity without altering the local statistics. This is similar 
to the convexification in the metastability problem, which does not alter events before the escape time.

2.3. Some details on the proof of Theorem 2.2. The core of the proof is divided into three theorems. For the flow with generator $\widetilde{\mathscr{L}}$, we have the following estimates on the entropy and Dirichlet form.

Theorem 2.3. Consider the forward equation

$$
\partial_{t} q_{t}=\widetilde{\mathscr{L}} q_{t}, \quad t \geq 0,
$$

with initial condition $q_{0}=q$ and with the reversible measure $\omega$. Assume that $\int q_{0} \mathrm{~d} \omega=1$. Then we have the estimates

$$
\begin{gathered}
\partial_{t} D_{\omega}\left(\sqrt{q_{t}}\right) \leq-\frac{1}{2 \tau} D_{\omega}\left(\sqrt{q_{t}}\right)-\frac{1}{2 N^{2}} \int \sum_{i, j=1}^{N} \frac{\left(\partial_{i} \sqrt{q_{t}}-\partial_{j} \sqrt{q_{t}}\right)^{2}}{\left(x_{i}-x_{j}\right)^{2}} \mathrm{~d} \omega, \\
\frac{1}{2 N^{2}} \int_{0}^{\infty} \mathrm{d} s \int \sum_{i, j=1}^{N} \frac{\left(\partial_{i} \sqrt{q_{s}}-\partial_{j} \sqrt{q_{s}}\right)^{2}}{\left(x_{i}-x_{j}\right)^{2}} \mathrm{~d} \omega \leq D_{\omega}(\sqrt{q}),
\end{gathered}
$$

and the logarithmic Sobolev inequality

$$
S_{\omega}(q) \leq C \tau D_{\omega}(\sqrt{q})
$$

with a universal constant $C$. Thus the relaxation time to equilibrium is of order $\tau$ :

$$
S_{\omega}\left(q_{t}\right) \leq e^{-C t / \tau} S_{\omega}(q) .
$$

Proof. Denote by $h=\sqrt{q}$, and we have the equation

$$
\partial_{t} D_{\omega}\left(h_{t}\right)=\partial_{t} \frac{1}{2 N} \int(\nabla h)^{2} e^{-N \widetilde{\mathcal{H}}} \mathrm{d} \mathbf{x} \leq-\frac{1}{2 N} \int \nabla h\left(\nabla^{2} \widetilde{\mathcal{H}}\right) \nabla h e^{-N \widetilde{\mathcal{H}}} \mathrm{d} \mathbf{x} .
$$

In our case, (2.22) and (2.23) imply that the Hessian of $\tilde{\mathcal{H}}$ is bounded from below as

$$
\nabla h\left(\nabla^{2} \tilde{\mathcal{H}}\right) \nabla h \geq \frac{C}{\tau} \sum_{j}\left(\partial_{j} h\right)^{2}+\frac{1}{2 N} \sum_{i, j} \frac{1}{\left(x_{i}-x_{j}\right)^{2}}\left(\partial_{i} h-\partial_{j} h\right)^{2}
$$

with some positive constant $C$. This proves (2.27) and (2.28). The rest can be proved by straightforward arguments given in the earlier part of this section.

The estimate (2.28) plays a key role in the next theorem.

Theorem 2.4 (Dirichlet form inequality). Let $q$ be a probability density $\int q \mathrm{~d} \omega=1$, and let $G: \mathbb{R} \rightarrow \mathbb{R}$ be a smooth function with compact support. Then for any $J \subset\{1,2, \ldots, N-1\}$ and any $t>0$, we have

$$
\begin{aligned}
& \left|\int \frac{1}{|J|} \sum_{i \in J} G\left(N\left(x_{i}-x_{i+1}\right)\right) q \mathrm{~d} \omega-\int \frac{1}{|J|} \sum_{i \in J} G\left(N\left(x_{i}-x_{i+1}\right)\right) \mathrm{d} \omega\right| \\
& \leq C\left(t \frac{D_{\omega}(\sqrt{q})}{|J|}\right)^{1 / 2}+C \sqrt{S_{\omega}(q)} e^{-c t / \tau} .
\end{aligned}
$$

Proof. For simplicity, we assume that $J=\{1,2, \ldots, N-1\}$. Let $q_{t}$ satisfy

$$
\partial_{t} q_{t}=\widetilde{\mathscr{L}} q_{t}, \quad t \geq 0
$$


with an initial condition $q_{0}=q$. We write

$$
\begin{aligned}
\int\left[\frac{1}{|J|} \sum_{i \in J} G\left(N\left(x_{i}-x_{i+1}\right)\right)\right](q-1) \mathrm{d} \omega \\
=\int\left[\frac{1}{|J|} \sum_{i \in J} G\left(N\left(x_{i}-x_{i+1}\right)\right)\right]\left(q-q_{t}\right) \mathrm{d} \omega \\
\quad+\int\left[\frac{1}{|J|} \sum_{i \in J} G\left(N\left(x_{i}-x_{i+1}\right)\right)\right]\left(q_{t}-1\right) \mathrm{d} \omega
\end{aligned}
$$

The second term can be estimated by (2.13), the decay of the entropy (2.30) and the boundedness of $G$; this gives the second term in (2.33).

To estimate the first term in (2.34), by the evolution equation $\partial q_{t}=\widetilde{\mathscr{L}} q_{t}$ and the definition of $\widetilde{\mathscr{L}}$,

$$
\begin{gathered}
\int \frac{1}{|J|} \sum_{i \in J} G\left(N\left(x_{i}-x_{i+1}\right)\right) q_{t} \mathrm{~d} \omega-\int \frac{1}{|J|} \sum_{i \in J} G\left(N\left(x_{i}-x_{i+1}\right)\right) q_{0} \mathrm{~d} \omega \\
=\int_{0}^{t} \mathrm{~d} s \int \frac{1}{|J|} \sum_{i \in J} G^{\prime}\left(N\left(x_{i}-x_{i+1}\right)\right)\left[\partial_{i} q_{s}-\partial_{i+1} q_{s}\right] \mathrm{d} \omega .
\end{gathered}
$$

From the Schwarz inequality and $\partial q=2 \sqrt{q} \partial \sqrt{q}$, the last term is bounded by

$$
\begin{aligned}
2\left[\int_{0}^{t} \mathrm{~d}\right. & \left.s \int_{\mathbb{R}^{N}} \frac{N^{2}}{|J|^{2}} \sum_{i \in J}\left[G^{\prime}\left(N\left(x_{i}-x_{i+1}\right)\right)\right]^{2}\left(x_{i}-x_{i+1}\right)^{2} q_{s} \mathrm{~d} \omega\right]^{1 / 2} \\
& \times\left[\int_{0}^{t} \mathrm{~d} s \int_{\mathbb{R}^{N}} \frac{1}{N^{2}} \sum_{i} \frac{1}{\left(x_{i}-x_{i+1}\right)^{2}}\left[\partial_{i} \sqrt{q_{s}}-\partial_{i+1} \sqrt{q_{s}}\right]^{2} \mathrm{~d} \omega\right]^{1 / 2} \\
\leq & C\left(\frac{D_{\omega}\left(\sqrt{q_{0}}\right) t}{|J|}\right)^{1 / 2}
\end{aligned}
$$

where we have used (2.28) and that $\left[G^{\prime}\left(N\left(x_{i}-x_{i+1}\right)\right)\right]^{2}\left(x_{i}-x_{i+1}\right)^{2} \leq C N^{-2}$ due to $G$ being smooth and compactly supported.

Alternatively, we could have directly estimated the left-hand side of (2.33) by using the total variation norm between $q \omega$ and $\omega$, which in turn could be estimated by the entropy (2.13) and the Dirichlet form using the logarithmic Sobolev inequality, i.e., by

$$
C \int|q-1| \mathrm{d} \omega \leq C \sqrt{S_{\omega}(q)} \leq C \sqrt{\tau D_{\omega}(\sqrt{q})} .
$$

However, compared with this simple bound, the estimate 2.33 gains an extra factor $|J| \sim N$ in the denominator, i.e., it is in terms of Dirichlet form per particle. The improvement is due to the observable in (2.33) being of special form and we exploit the term (2.28).

The final ingredient in proving Theorem 2.2 is the following entropy and Dirichlet form estimates.

Theorem 2.5. Suppose that (2.22) holds. Let $\mathfrak{a}>0$ be fixed, and recall the definition of $Q=Q_{\mathfrak{a}}$ from (2.5). Fix a constant $\tau \geq N^{-2 \mathfrak{a}}$ and consider the local 
relaxation measure $\omega$ with this $\tau$. Set $\psi:=\omega / \mu$, and let $g_{t}:=f_{t} / \psi$. Suppose there is a constant $m$ such that

$$
S\left(f_{\tau} \omega \mid \omega\right) \leq C N^{m}
$$

Then for any $t \geq \tau N^{\varepsilon}$ the entropy and the Dirichlet form satisfy the estimates,

$$
S\left(g_{t} \omega \mid \omega\right) \leq C N^{2} Q \tau^{-1}, \quad D_{\omega}\left(\sqrt{g_{t}}\right) \leq C N^{2} Q \tau^{-2},
$$

where the constants depend on $\varepsilon$ and $m$.

Proof. The evolution of the entropy $S\left(f_{t} \mu \mid \omega\right)=S_{\omega}\left(g_{t}\right)$ can be computed explicitly by the formula $(78]$ )

$$
\partial_{t} S\left(f_{t} \mu \mid \omega\right)=-\frac{2}{N} \sum_{j} \int\left(\partial_{j} \sqrt{g_{t}}\right)^{2} \psi \mathrm{d} \mu+\int g_{t} \mathscr{L} \psi \mathrm{d} \mu .
$$

Hence by using (2.25) we have

$$
\partial_{t} S\left(f_{t} \mu \mid \omega\right)=-\frac{2}{N} \sum_{j} \int\left(\partial_{j} \sqrt{g_{t}}\right)^{2} \mathrm{~d} \omega+\int \widetilde{\mathscr{L}} g_{t} \mathrm{~d} \omega+\sum_{j} \int b_{j} \partial_{j} g_{t} \mathrm{~d} \omega .
$$

Since $\omega$ is $\widetilde{\mathscr{L}}$-invariant and time independent, the middle term on the right-hand side vanishes, and from the Schwarz inequality

$$
\partial_{t} S\left(f_{t} \mu \mid \omega\right) \leq-D_{\omega}\left(\sqrt{g_{t}}\right)+C N \sum_{j} \int b_{j}^{2} g_{t} \mathrm{~d} \omega \leq-D_{\omega}\left(\sqrt{g_{t}}\right)+C N^{2} Q \tau^{-2} .
$$

Together with the logarithmic Sobolev inequality (2.29), we have

$$
\partial_{t} S\left(f_{t} \mu \mid \omega\right) \leq-D_{\omega}\left(\sqrt{g_{t}}\right)+C N^{2} Q \tau^{-2} \leq-C \tau^{-1} S\left(f_{t} \mu \mid \omega\right)+C N^{2} Q \tau^{-2} .
$$

Integrating the last inequality from $\tau$ to $t$ and using the assumption (2.37) and $t \geq \tau N^{\varepsilon}$, we have proved the first inequality of (2.38). Using this result and integrating (2.39), we have

$$
\int_{\tau}^{t} D_{\omega}\left(\sqrt{g_{s}}\right) \mathrm{d} s \leq C N^{2} Q \tau^{-1}
$$

By the convexity of the Hamiltonian, $D_{\mu}\left(\sqrt{f_{t}}\right)$ is decreasing in $t$. Since $D_{\omega}\left(\sqrt{g_{s}}\right) \leq$ $C D_{\mu}\left(\sqrt{f_{s}}\right)+C N^{2} Q \tau^{-2}$, this proves the second inequality of (2.38).

Finally, we complete the proof of Theorem 2.2. For any given $t>0$, we now choose $\tau:=t N^{-\varepsilon}$, and we construct the local relaxation measure $\omega$ with this $\tau$. Set $\psi=\omega / \mu$, and let $q:=g_{t}=f_{t} / \psi$ be the density $q$ in Theorem 2.4. Then Theorem 2.5. Theorem 2.4 and an easy bound on the entropy $S_{\omega}(q) \leq C N^{m}$ imply that

$$
\begin{gathered}
\left|\int \frac{1}{N} \sum_{i \in J} G\left(N\left(x_{i}-x_{i+1}\right)\right)\left(f_{t} \mathrm{~d} \mu-\mathrm{d} \omega\right)\right| \leq C\left(t \frac{D_{\omega}(\sqrt{q})}{|J|}\right)^{1 / 2}+C \sqrt{S_{\omega}(q)} e^{-c N^{\varepsilon}} \\
\leq C\left(t \frac{N^{2} Q}{|J| \tau^{2}}\right)^{1 / 2}+C e^{-c N^{\varepsilon}} \leq C N^{\varepsilon} \sqrt{\frac{N^{2} Q}{|J| t}}+C e^{-c N^{\varepsilon}}
\end{gathered}
$$

i.e., the local statistics of $f_{t} \mu$ and $\omega$ are the same for any initial data $f_{\tau}$ for which (2.37) is satisfied. Applying the same argument to the Gaussian initial data, $f_{0}=$ $f_{\tau}=1$, we can also compare $\mu$ and $\omega$. We have thus proved (2.9) and hence the universality. 


\section{Local SEMicircle LAW Via Green FunCtion}

The Wigner semicircle law asserts that (1.2) is valid in a weak limit, i.e., for any smooth test function $O$ with compact support, we have

$$
\mathbb{E} \int_{\mathbb{R}} O(x)\left[\varrho_{N}(x)-\varrho_{s c}(x)\right] \mathrm{d} x \rightarrow 0 .
$$

This means that the density of eigenvalues in a window independent of $N$ is given by the semicircle law. Our goal is to prove a local version of this result for windows slightly larger than $1 / N$ and in a large deviation sense. The main object to study is the Green function of the matrix $G(z)=[H-z]^{-1}, z=E+i \eta, E \in \mathbb{R}, \eta>0$, which is related to the Stieltjes transform of the empirical measure,

$$
m(z)=m_{N}(z):=\frac{1}{N} \operatorname{Tr} \frac{1}{H-z}=\frac{1}{N} \sum_{j=1}^{N} \frac{1}{\lambda_{j}-z}=\int_{\mathbb{R}} \frac{\mathrm{d} \varrho_{N}(x)}{x-z}=\frac{1}{N} \sum_{j=1}^{N} G_{j j}(z) .
$$

We will compare it with $m_{s c}(z):=\int_{\mathbb{R}}(x-z)^{-1} \varrho_{s c}(x) \mathrm{d} x$, the Stieltjes transform of the semicircle law. This is the content of the local semicircle law, Theorem 3.1 below. The key parameter is $\eta=\mathfrak{I m} z$ which determines the resolution, i.e., the scale on which the local semicircle law holds.

For the rest of this paper, we will assume that the probability distribution of the matrix elements satisfy the subexponential condition,

$$
\mathbb{P}\left(\left|v_{i j}\right| \geq x\right) \leq C_{0} \exp \left(-x^{\vartheta}\right), \quad x>0,
$$

with some positive constants $C_{0}, \vartheta$, where we set $v_{i j}=\sqrt{N} h_{i j}$. This condition can be relaxed to (1.8) via a cutoff argument, but we will not discuss such technical details here.

Theorem 3.1 (Local semicircle law [38, Theorem 2.1]). Let $H=\left(h_{i j}\right)$ be a Hermitian or symmetric $N \times N$ random matrix with $\mathbb{E} h_{i j}=0,1 \leq i, j \leq N$. Suppose that the distributions of the matrix elements have a uniformly subexponential decay (3.3). Then there exist positive constants $A_{0}>1, C, c$ and $\phi<1$ such that with

$$
L:=A_{0} \log \log N
$$

the following estimates hold for any sufficiently large $N \geq N_{0}\left(C_{0}, \vartheta\right)$.

(i) The Stieltjes transform of the empirical eigenvalue distribution of $H$ satisfies

$$
\mathbb{P}\left(\bigcup_{z \in \mathbf{S}_{L}}\left\{\left|m(z)-m_{s c}(z)\right| \geq \frac{(\log N)^{4 L}}{N \eta}\right\}\right) \leq C \exp \left[-c(\log N)^{\phi L}\right],
$$

where

$$
\mathbf{S}_{L}:=\left\{z=E+i \eta:|E| \leq 5, \quad N^{-1}(\log N)^{10 L}<\eta \leq 10\right\} .
$$

(ii) The individual matrix elements of the Green function satisfy

$$
\begin{aligned}
\mathbb{P}\left(\bigcup_{z \in \mathbf{S}_{L}}\right. & \left.\left\{\max _{i, j}\left|G_{i j}(z)-\delta_{i j} m_{s c}(z)\right| \geq(\log N)^{4 L} \sqrt{\frac{\mathfrak{I m} m_{s c}(z)}{N \eta}}+\frac{(\log N)^{4 L}}{N \eta}\right\}\right) \\
\leq & C \exp \left[-c(\log N)^{\phi L}\right] .
\end{aligned}
$$


Theorem 3.1 is the strongest form of the local semicircle law that gives optimal error estimates (modulo logarithmic factors) on the smallest possible scale, which is valid uniformly in the spectrum including the edge, and which controls not only the Stieltjes transform but also individual matrix elements of the resolvent. This theorem is the final result of subsequent improvements in [31, 32, 36, 37, 38, of our first local semicirle law in 30 .

The local semicircle estimates imply that the $j$-th eigenvalue, $\lambda_{j}$, is very close to its classical location $\gamma_{j}$, defined in (2.4):

Corollary 3.2 (Rigidity of eigenvalues [38, Theorem 2.2]). Under the assumptions of Theorem 3.1 we have

$$
\begin{aligned}
\mathbb{P}\{\exists j: & \left.\left|\lambda_{j}-\gamma_{j}\right| \geq(\log N)^{L}[\min (j, N-j+1)]^{-1 / 3} N^{-2 / 3}\right\} \\
& \leq C \exp \left[-c(\log N)^{\phi L}\right]
\end{aligned}
$$

for any sufficiently large $N \geq N_{0}$.

This corollary in particular proves the a priori estimate (2.5) for any $\mathfrak{a}<1 / 2$.

Corollary 3.2 is a simple consequence of the Helffer-Sjöstrand formula which translates information on the Stieltjes transform of the empirical measure first to the counting function and then to the locations of eigenvalues. The formula yields the representation

$$
\begin{aligned}
f(\lambda) & =\frac{1}{2 \pi} \int_{\mathbb{R}^{2}} \frac{\partial_{\bar{z}} \tilde{f}(x+i y)}{\lambda-x-i y} \mathrm{~d} x \mathrm{~d} y \\
& =\frac{1}{2 \pi} \int_{\mathbb{R}^{2}} \frac{i y f^{\prime \prime}(x) \chi(y)+i\left(f(x)+i y f^{\prime}(x)\right) \chi^{\prime}(y)}{\lambda-x-i y} \mathrm{~d} x \mathrm{~d} y
\end{aligned}
$$

for any real valued $C^{2}$ function $f$ on $\mathbb{R}$, where $\chi(y)$ is any smooth cutoff function with bounded derivatives and supported in $[-1,1]$ with $\chi(y)=1$ for $|y| \leq 1 / 2$. In the applications, $f$ will be a smoothed version of the characteristic functions of spectral intervals so that $\sum_{j} f\left(\lambda_{j}\right)$ counts eigenvalues in that interval. The details of the argument can be found in 29].

We also mention that Theorem 3.1 immediately implies complete delocalization of each eigenvector of the Wigner matrix:

Corollary 3.3 (Complete delocalization). Let $u_{1}, u_{2}, \ldots$ be the $\ell^{2}$-normalized eigenvectors of $H$. Under the assumptions of Theorem 3.1 we have

$$
\mathbb{P}\left\{\exists \beta:\left\|u_{\beta}\right\|_{\infty}^{2} \geq \frac{(\log N)^{10 L}}{N}\right\} \leq C \exp \left[-c(\log N)^{\phi L}\right]
$$

for any sufficiently large $N \geq N_{0}$.

For the proof, notice that (3.7) implies the bound $\left|G_{j j}(z)\right|=O(1)$ with very high probability for any $z \in \mathbf{S}_{L}$. Therefore,

$$
C \geq \mathfrak{I m} G_{j j}\left(\lambda_{\alpha}+i \eta\right)=\sum_{\beta} \frac{\eta\left|u_{\beta}(j)\right|^{2}}{\left(\lambda_{\beta}-\lambda_{\alpha}\right)^{2}+\eta^{2}} \geq \frac{\left|u_{\alpha}(j)\right|^{2}}{\eta} .
$$

The original proof of delocalization of eigenvectors was derived from the Stieltjes transform of the empirical measure [30, 32, motivated by a question posed by $\mathrm{T}$. Spencer. 
Sketch of the proof of Theorem 3.1. For simplicity, we will assume here that $E=$ $\mathfrak{R e} z$ is away from the spectral edges. The starting point is the following well known formula. Let $A, B, C$ be $n \times n, m \times n$ and $m \times m$ matrices, and set

$$
D:=\left(\begin{array}{cc}
A & B^{*} \\
B & C
\end{array}\right) \text {. }
$$

Then for any $1 \leq i, j \leq n$, we have

$$
\left(D^{-1}\right)_{i j}=\left[\left(A-B^{*} C^{-1} B\right)^{-1}\right]_{i j} .
$$

Applying this formula to the resolvent matrix $G=(H-z)^{-1}$, we have

$$
G_{i i}=\frac{1}{h_{i i}-z-\sum_{k, l \neq i} h_{i k} G_{k l}^{(i)} h_{l i}}=\frac{1}{h_{i i}-z-\mathbb{E}_{i} \sum_{k, l \neq i} h_{i k} G_{k l}^{(i)} h_{l i}-Z_{i}},
$$

where

$$
Z_{i}:=\sum_{k, l \neq i} h_{i k} G_{k l}^{(i)} h_{l i}-\sum_{k, l \neq i} \mathbb{E}_{i} h_{i k} G_{k l}^{(i)} h_{l i}
$$

Here $G^{(i)}$ denotes the resolvent of the $(N-1) \times(N-1)$ minor of $H$ after removing the $i$-th row and column and $\mathbb{E}_{i}$ denotes the expectation with respect to the entries in the $i$-th row and column. Since $G^{(i)}$ is independent of $h_{i k}$ and $\mathbb{E}_{i} h_{i k} h_{l i}=\frac{1}{N} \delta_{k l}$, we have

$$
\sum_{k, l \neq i} \mathbb{E}_{i} h_{i k} G_{k l}^{(i)} h_{l i}=\frac{1}{N} \sum_{k \neq i} G_{k k}^{(i)}=\frac{1}{N} \sum_{k} G_{k k}+O\left(\frac{1}{N}\right) .
$$

Here we used the interlacing property of eigenvalues between a matrix and its minors, which implies that

$$
\left|\frac{1}{N} \operatorname{Tr} G-\frac{1}{N} \operatorname{Tr} G^{(i)}\right|=\left|m(z)-m^{(i)}(z)\right| \leq \frac{C}{N \eta}, \quad \eta=\mathfrak{I m} z>0 .
$$

Defining $v_{i}:=G_{i i}-m_{s c}$, we thus have

$$
v_{i}=G_{i i}-m_{s c}=\frac{1}{-z-m_{s c}-\left(\frac{1}{N} \sum_{j} v_{j}+Z_{i}-h_{i i}+O\left(N^{-1}\right)\right)}-m_{s c} .
$$

Expanding the denominator, using the identity $m_{s c}(z)+\left[m_{s c}(z)+z\right]^{-1}=0$ and neglecting the error terms $h_{i i}+O\left(N^{-1}\right)=O\left(N^{-1 / 2}\right)$, we have

$$
v_{i}=m_{s c}^{2}\left(\frac{1}{N} \sum_{j} v_{j}+Z_{i}\right)+m_{s c}^{3}\left(\frac{1}{N} \sum_{j} v_{j}+Z_{i}\right)^{2}+\cdots .
$$

Summing up $i$ and dividing by $N$, we obtain, modulo negligible errors,

$$
[v]:=\frac{1}{N} \sum_{j} v_{j} \approx m_{s c}^{2}[v]+m_{s c}^{3}[v]^{2}+m_{s c}^{2}[Z]+O\left(\frac{1}{N} \sum_{i}\left|Z_{i}\right|^{2}\right), \quad[Z]:=\frac{1}{N} \sum_{j} Z_{j} .
$$

To estimate $Z_{i}$, we compute its second moment

$$
\begin{aligned}
\mathbb{E}\left|Z_{i}\right|^{2}= & \mathbb{E} \sum_{k, l \neq i} \sum_{k^{\prime}, l^{\prime} \neq i} \\
& \times \mathbb{E}_{i}\left(\left[h_{i k} G_{k l}^{(i)} h_{l i}-\mathbb{E}_{i} h_{i k} G_{k l}^{(i)} h_{l i}\right]\left[\bar{h}_{i k^{\prime}} \bar{G}_{k^{\prime} l^{\prime}}^{(i)} \bar{h}_{l^{\prime} i}-\mathbb{E}_{i} \bar{h}_{i k^{\prime}} \bar{G}_{k^{\prime} l^{\prime}}^{(i)} \bar{h}_{l^{\prime} i}\right]\right) .
\end{aligned}
$$


Since $\mathbb{E} h=0$, the non-zero contributions to this sum come from index combinations when all $h$ and $\bar{h}$ are paired. For pedagogical simplicity, assume that $\mathbb{E} h^{2}=0$, this can be achieved, for example, if the distribution of the real and imaginary parts are the same. Then the $h$ factors in the above expression have to be paired in such a way that $h_{i k}=h_{i k^{\prime}}$ and $h_{i l}=h_{i l^{\prime}}$, i.e., $k=k^{\prime}, l=l^{\prime}$. Note that pairing $h_{i k}=h_{i l}$ would give zero because the expectation is subtracted. The result is

$$
\mathbb{E}_{i}\left|Z_{i}\right|^{2}=\frac{1}{N^{2}} \sum_{k, l \neq i}\left|G_{k l}^{(i)}\right|^{2}+\frac{m_{4}-1}{N^{2}} \sum_{k \neq i}\left|G_{k k}^{(i)}\right|^{2},
$$

where $m_{4}=\mathbb{E}|\sqrt{N} h|^{4}$ is the fourth moment of the single entry distribution. The first term can be computed

$$
\frac{1}{N^{2}} \sum_{k, l \neq i}\left|G_{k l}^{(i)}\right|^{2}=\frac{1}{N^{2}} \sum_{k \neq i}\left(\left|G^{(i)}\right|^{2}\right)_{k k}=\frac{1}{N \eta} \frac{1}{N} \sum_{k} \mathfrak{I m} G_{k k}^{(i)}:=\frac{1}{N \eta} \mathfrak{I m} m^{(i)}
$$

The second term in (3.20) can be estimated by a similar bound. These estimates confirm that the size of $Z_{i}$, at least in the second moment sense, is roughly

$$
\left|Z_{i}\right| \lesssim \frac{C}{\sqrt{N \eta}}
$$

Neglecting the $[v]^{2}$ term in (3.18) and using that $\left|1-m_{s c}^{2}\right| \geq c$ away from the spectral edge for some positive $c$, we thus have $\left|m(z)-m_{s c}(z)\right| \lesssim C(N \eta)^{-1 / 2}$. A similar but more involved argument gives the same bound for individual $v_{i}$ 's, showing the estimate (3.7) for the diagonal elements $G_{i i}$. The estimate for the offdiagonal terms, $G_{i j}, i \neq j$, is obtained from the identity $G_{i j}=G_{j j} G_{i i}^{(j)}\left[Z_{i j}-h_{i j}\right]$ which can be proved using (3.12). Here $Z_{i j}$ is defined analogously to (3.14) as

$$
Z_{i j}:=\sum_{k, l \neq i, j} h_{i k} G_{k l}^{(i j)} h_{l j}-\sum_{k, l \neq i, j} \mathbb{E}_{i j} h_{i k} G_{k l}^{(i j)} h_{l j},
$$

where $G^{(i j)}$ is the resolvent of the $(N-2) \times(N-2)$ minor of $H$ after removing the $i$-th and $j$-th row and column. The bound (3.22) holds for $Z_{i j}$ as well.

The estimate for $[v]=m-m_{s c}$, the average of $v_{i}$ 's, is of order $(N \eta)^{-1}$ in (3.5), i.e., it is better than the $(N \eta)^{-1 / 2}$ estimate for the individual matrix elements in (3.7). The key mechanism for this improvement is the cancellation of the $Z_{j}$ 's in their average [Z]. If $Z_{j}$ 's were independent, we would gain a factor $N^{-1 / 2}$ by the central limit theorem. But $Z_{j}$ 's are correlated and the cancellation takes the following form:

Lemma 3.4 (Fluctuation Averaging Lemma). With the notations of Theorem 3.1, for any $\varepsilon>0$ we have

$$
\mathbb{P}\left(\frac{1}{N}\left|\sum_{i=1}^{N} Z_{i}\right| \geq \frac{N^{\varepsilon}}{N \eta}\right) \leq C \exp \left[-c(\log N)^{\phi L}\right]
$$

for sufficiently large $N$.

Using this lemma and (3.18), we have proved the stronger estimate for $[v]$. This completes the sketch of the proof of the local semicircle law, Theorem 3.1 


\section{The Green function COMPARIson theorems}

We now state the Green function comparison theorem, Theorem 4.1. It will quickly lead to Theorem 4.2 stating that the correlation functions of eigenvalues of two matrix ensembles are identical on a scale smaller than $1 / N$ provided that the first four moments of all matrix elements of these two ensembles are almost the same. We will state a limited version for real Wigner matrices for simplicity of presentation.

Theorem 4.1 (Green function comparison [36, Theorem 2.3]). Suppose that we have two $N \times N$ Wigner matrices, $H^{(v)}$ and $H^{(w)}$, with matrix elements $h_{i j}$ given by the random variables $N^{-1 / 2} v_{i j}$ and $N^{-1 / 2} w_{i j}$, respectively, with $v_{i j}$ and $w_{i j}$ satisfying the uniform subexponential decay condition (3.3). We assume that the first four moments of $v_{i j}$ and $w_{i j}$ are close to each other in the sense that

$$
\left|\mathbb{E} v_{i j}^{s}-\mathbb{E} w_{i j}^{s}\right| \leq N^{-\delta-2+s / 2}, \quad 1 \leq s \leq 4,
$$

holds for some $\delta>0$. Then there are positive constants $C_{1}$ and $\varepsilon$, depending on $\vartheta$ and $C_{0}$ from (3.3) such that for any $\eta$ with $N^{-1-\varepsilon} \leq \eta \leq N^{-1}$ and for any $z_{1}, z_{2}$ with $\mathfrak{I m} z_{j}= \pm \eta, j=1,2$, we have

$$
\lim _{N \rightarrow \infty}\left[\mathbb{E} \operatorname{Tr} G^{(v)}\left(z_{1}\right) \operatorname{Tr} G^{(v)}\left(z_{2}\right)-\mathbb{E} \operatorname{Tr} G^{(w)}\left(z_{1}\right) \operatorname{Tr} G^{(w)}\left(z_{2}\right)\right]=0,
$$

where $G^{(v)}$ and $G^{(w)}$ denote the Green functions of $H^{(v)}$ and $H^{(w)}$.

The matching condition in (4.1) is essentially the same as the one that appeared in 67. Here we formulated Theorem 4.1 for a product of two traces of the Green function, but the result holds for a large class of smooth functions depending on several individual matrix elements of the Green functions as well; see [36] for the precise statement. (The matching condition (4.1) is slightly weaker than in 36, but the proof in [36] without any change yields this slightly stronger version.) This general version of Theorem 4.1 implies the correlation functions of the two ensembles at the scale $1 / N$ are identical:

Theorem 4.2 (Correlation function comparison [36, Theorem 6.4]). Suppose the assumptions of Theorem 4.1 hold. Let $p_{v, N}^{(n)}$ and $p_{w, N}^{(n)}$ be the n-point functions of the eigenvalues w.r.t. the probability law of the matrix $H^{(v)}$ and $H^{(w)}$, respectively. Then for any $|E|<2$, any $n \geq 1$, and any compactly supported continuous test function $O: \mathbb{R}^{n} \rightarrow \mathbb{R}$, we have

$$
\lim _{N \rightarrow \infty} \int_{\mathbb{R}^{n}} \mathrm{~d} \alpha_{1} \cdots \mathrm{d} \alpha_{n} O\left(\alpha_{1}, \ldots, \alpha_{n}\right)\left(p_{v, N}^{(n)}-p_{w, N}^{(n)}\right)\left(E+\frac{\alpha_{1}}{N}, \ldots, E+\frac{\alpha_{n}}{N}\right)=0 .
$$

The basic idea for proving Theorem 4.1 is similar to Lindeberg's proof of the central limit theorem, where the random variables are replaced one by one with a Gaussian one. We will replace the matrix elements $v_{i j}$ with $w_{i j}$ one by one and estimate the effect of this change on the resolvent by a resolvent expansion. The idea of applying Lindeberg's method in random matrices was recently used by Chatterjee [13] for comparing the traces of the Green functions; the idea was also used by Tao and $\mathrm{Vu}$ [67. in the context of comparing individual eigenvalue distributions. There are two main differences between our method and the one that appeared in [67]: 
(i) We compare the statistics of eigenvalues of two different ensembles near fixed energies, while 67] compared the statistics of the $j_{1}, j_{2}, \ldots, j_{k}$-th eigenvalues for fixed labels $j_{1}, j_{2}, \ldots, j_{k}$.

(ii) There is a serious difficulty in the approach 67. concerning possible resonances of neighboring eigenvalues that may render the expansion unstable.

The Green function method eliminates this difficulty completely and Theorem 4.1 is a simple corollary of the Green function estimate Theorem 3.1

For a sketch of the proof, fix a bijective ordering map on the index set of the independent matrix elements,

$$
\phi:\{(i, j): 1 \leq i \leq j \leq N\} \rightarrow\{1, \ldots, \gamma(N)\}, \quad \gamma(N):=\frac{N(N+1)}{2},
$$

and denote by $H_{\gamma}$ the Wigner matrix whose matrix elements $h_{i j}$ follow the $v$ distribution if $\phi(i, j) \leq \gamma$ and they follow the $w$-distribution otherwise; in particular $H^{(v)}=H_{0}$ and $H^{(w)}=H_{\gamma(N)}$.

Consider the telescopic sum of differences of expectations (we present only one resolvent for simplicity of presentation):

$$
\begin{aligned}
& \mathbb{E}(\left.\frac{1}{N} \operatorname{Tr} \frac{1}{H^{(w)}-z}\right)-\mathbb{E}\left(\frac{1}{N} \operatorname{Tr} \frac{1}{H^{(v)}-z}\right) \\
& \quad=\sum_{\gamma=1}^{\gamma(N)}\left[\mathbb{E}\left(\frac{1}{N} \operatorname{Tr} \frac{1}{H_{\gamma}-z}\right)-\mathbb{E}\left(\frac{1}{N} \operatorname{Tr} \frac{1}{H_{\gamma-1}-z}\right)\right] .
\end{aligned}
$$

Let $E^{(i j)}$ denote the matrix whose matrix elements are zero everywhere except at the $(i, j)$ position, where it is 1 , i.e., $E_{k \ell}^{(i j)}=\delta_{i k} \delta_{j \ell}$. Fix a $\gamma \geq 1$, and let $(i, j)$ be determined by $\phi(i, j)=\gamma$. We will compare $H_{\gamma-1}$ with $H_{\gamma}$. Note that these two matrices differ only in the $(i, j)$ and $(j, i)$ matrix elements and they can be written as

$$
\begin{aligned}
& H_{\gamma-1}=Q+\frac{1}{\sqrt{N}} V, \quad V:=v_{i j} E^{(i j)}+v_{j i} E^{(j i)}, \quad v_{j i}:=\bar{v}_{i j}, \\
& H_{\gamma}=Q+\frac{1}{\sqrt{N}} W, \quad W:=w_{i j} E^{(i j)}+w_{j i} E^{(j i)}, \quad w_{j i}:=\bar{w}_{i j},
\end{aligned}
$$

with a matrix $Q$ that has zero matrix element at the $(i, j)$ and $(j, i)$ positions.

By the resolvent expansion,

$$
\begin{aligned}
S_{\gamma-1}=R-N^{-1 / 2} R V R+\cdots+N^{-2}(R V)^{4} R-N^{-5 / 2}(R V)^{5} S & \\
R & :=\frac{1}{Q-z}, \quad S_{\gamma-1}:=\frac{1}{H_{\gamma-1}-z},
\end{aligned}
$$

and a similar expression holds for the resolvent $S_{\gamma}$ of by $H_{\gamma}$. From the local semicircle law for individual matrix elements (3.7), the matrix elements of all Green functions $R, S_{\gamma-1}, S_{\gamma}$ are bounded by $C N^{\varepsilon}$ for any $\varepsilon>0$. By assumption (4.1), the difference between the expectation of matrix elements of $S_{\gamma-1}$ and $S_{\gamma}$ is of order $N^{-2-\delta+C \varepsilon}$. Since the number of steps, $\gamma(N)$ is of order $N^{2}$, the difference in (4.4) is of order $N^{2} N^{-2-\delta+C \varepsilon} \ll 1$, and this proves Theorem 4.1 for a single resolvent. It is very simple to turn this heuristic argument into a rigorous proof and to generalize it to the product of several resolvents. The real difficulty is the input that the local semicircle law holds for a general class of Wigner matrices. 


\section{Universality for Wigner matrices: PUtting it together}

In this short section we put the previous information together to prove Theorem 1.1. We first focus on the case when $b_{N}$ is independent of $N$. Recall that Theorem 2.1 states that the correlation functions of the Gaussian divisible ensemble,

$$
H_{t}=e^{-t / 2} H_{0}+\left(1-e^{-t}\right)^{1 / 2} U,
$$

where $H_{0}$ is the initial Wigner matrix and $U$ is an independent standard GUE (or GOE) matrix, are given by the corresponding GUE (or GOE) for $t \geq N^{-2 \mathfrak{a}+\varepsilon}$ provided that the a priori estimate (2.5) holds for the solution $f_{t}$ of the forward equation (2.2) with some exponent $\mathfrak{a}>0$. Since the rigidity of eigenvalues, Corollary 3.2 . holds uniformly for all Wigner matrices, we have proved (2.5) for $\mathfrak{a}=1 / 2-\varepsilon$ with any $\varepsilon>0$.

From the evolution of the OU process (2.1) for $v_{i j}=N^{1 / 2} h_{i j}$, we have

$$
\left|\mathbb{E} v_{i j}^{s}(t)-\mathbb{E} v_{i j}^{s}(0)\right| \leq C t=C N^{-1+3 \varepsilon}
$$

for $s=3,4$ and with the choice of $t=N^{-1+3 \varepsilon}$. Furthermore, $\mathbb{E} h_{i j}^{s}(t)$ are independent of $t$ for $s=1,2$ due to $\mathbb{E} v_{i j}(0)=0$ and $\mathbb{E} v_{i j}^{2}(t)=1$. Hence (4.1) is satisfied for the matrix elements of $H_{t}$ and $H_{0}$ and we can thus use Theorem 4.2 to conclude that the correlation functions of $H_{t}$ and $H_{0}$ are identical at the scale $1 / N$. Since the correlation functions of $H_{t}$ are given by the corresponding Gaussian case, we have proved Theorem 1.1 under the condition that the probability distribution of the matrix elements decay subexponentially. Finally, we need a technical cutoff argument to relax the decay condition, which we omit here (see Section 7 in [26]).

The argument for $N$-dependent $b=b_{N}$ in the range $b_{N} \geq N^{-1+\xi}, \xi>0$, is slightly different. For such a small $b_{N},(2.8)$ could be established only for relatively large times, $t \geq N^{-\xi / 8}$. We cannot therefore compare $H_{0}$ with $H_{t}$ directly, since the deviation of the third moments of $v_{i j}(0)$ and $v_{i j}(t)$ in (5.2) would not satisfy (4.11). Instead, we construct an auxiliary Wigner matrix $\widehat{H}_{0}$ such that up to the third moment its time evolution $\widehat{H}_{t}$ under the OU flow (5.1) matches exactly the original matrix $H_{0}$, and the fourth moments are close even for $t$ of order $N^{-\xi / 8}$ (see Lemma 3.4 of [37]). Theorem 2.1 will then be applied for $\widehat{H}_{t}$, and Theorem 4.1] can be used to compare $\widehat{H}_{t}$ and $H_{0}$.

We finally discuss the extension of Theorem 1.1 without averaging in $E^{\prime}$. For Hermitian matrices, with the notations of Theorem 1.1. for any fixed $|E|<2$ we have that

$$
\begin{aligned}
\int_{\mathbb{R}^{n}} \mathrm{~d} \alpha_{1} & \cdots \mathrm{d} \alpha_{n} O\left(\alpha_{1}, \ldots, \alpha_{n}\right) \frac{1}{\varrho_{s c}(E)^{n}} \\
& \times\left(p_{N}^{(n)}-p_{\mathrm{G}, N}^{(n)}\right)\left(E+\frac{\alpha_{1}}{N \varrho_{s c}(E)}, \ldots, E+\frac{\alpha_{n}}{N \varrho_{s c}(E)}\right)=0 .
\end{aligned}
$$

This convergence was first proved in Theorem 1.1 of [27] for matrices with distribution which is $C n$-times differentiable for some universal constant $C$. For a general distribution it was stated as Theorem 5 in [71. Although the proof in [71] took a slightly different path, this generalization is an immediate corollary of our previous results [35]. Recall our three step approach reviewed in the introduction. If we substitute Step $2 \mathrm{~b}$ with Step 2a, then all our results in the Hermitian case would need no time average. More precisely, Proposition 3.1 of [27] asserts that the bulk universality in the Hermitian case holds at a fixed energy for the Gaussian 
convolution matrix $H_{t}$ with $t \sim N^{-1+\delta}$. The first four moments of $H_{t}$ and $H_{0}$ are sufficiently close to apply directly the Green function comparison theorem for correlation functions (Theorem 4.2 in this article). This concludes the bulk universality of the original matrix $H_{0}$ at a fixed energy, which is the Theorem 5 in [71. In fact, our theory implies the same result for generalized Hermitian matrices (defined in Section 8) with finite $4+\varepsilon$ moments.

\section{Beta ensemble: Rigidity estimates}

The general $\beta$-ensemble with a potential $V$ is defined by the probability measure $\mu=\mu_{\beta, V}^{(N)}$ (1.7) on $N$ ordered real points $\lambda_{1} \leq \cdots \leq \lambda_{N}$. We let $\mathbb{P}_{\mu}$ and $\mathbb{E}_{\mu}$ denote the probability and the expectation with respect to $\mu$. For simplicity of presentation, we assume that the potential $V$ is convex, i.e.,

$$
\vartheta:=\frac{1}{2} \inf _{x \in \mathbb{R}} V^{\prime \prime}(x)>0,
$$

the equilibrium density $\varrho(s)$ is supported on a single interval $[A, B] \subset \mathbb{R}$ and satisfies (1.10) (for the general case, see [11]). The Gaussian case corresponds to $V(x)=$ $x^{2} / 2$, in which case the equilibrium density is the semicircle law, $\varrho_{s c}$, given by (1.2). Our main result concerning the universality is Theorem 1.2 and similar statement holds for the universality of the gap distributions directly. In fact, the proof of Theorem 1.2 goes via the gap distribution as we now explain.

Similarly to (2.4) we again denote by $\gamma_{k}$ the classical location of the $k$-th point w.r.t. the limiting equilibrium density $\varrho(s)$, i.e., $\gamma_{k}$ is defined by

$$
\int_{-\infty}^{\gamma_{k}} \varrho(s) \mathrm{d} s=\frac{k}{N} \text {. }
$$

The first step in proving Theorem 1.2 is the following theorem which provides a rigidity estimate on the location of each individual point in the bulk almost down to the optimal scale $1 / N$. In the following, we will denote $\llbracket x, y \rrbracket=\mathbb{N} \cap[x, y]$.

Theorem 6.1 ([10, Theorem 3.1]). Fix any $\alpha, \varepsilon>0$, and assume that (6.1) holds. Then there are constants $\delta, c_{1}, c_{2}>0$ such that for any $N \geq 1$ and $k \in$ $\llbracket \alpha N,(1-\alpha) N \rrbracket$,

$$
\mathbb{P}_{\mu}\left(\left|\lambda_{k}-\gamma_{k}\right|>N^{-1+\varepsilon}\right) \leq c_{1} e^{-c_{2} N^{\delta}} .
$$

The first ingredient in proving Theorem 6.1 is an analysis of the loop equation following Johansson [47] and Shcherbina 61]. The equilibrium density $\varrho$, for a convex potential $V$, is given by

$$
\varrho(t)=\frac{1}{\pi} r(t) \sqrt{(t-A)(B-t)} \mathbb{1}_{[A, B]}(t),
$$

where $r$ is a real function that can be extended to an analytic function in $\mathbb{C}$ and $r$ has no zero in $\mathbb{R}$. Denote by $s(z):=-2 r(z) \sqrt{(A-z)(B-z)}$ where the square root is defined such that its asymptotic value is $z$ as $z \rightarrow \infty$. Recall that the density is the one point correlation function which is characterized by

$$
\int_{\mathbb{R}} \mathrm{d} \lambda_{1} O\left(\lambda_{1}\right) p_{N}^{(1)}\left(\lambda_{1}\right)=\int_{\mathbb{R}^{N}} O\left(\lambda_{1}\right) \mathrm{d} \mu_{\beta, V}^{(N)}(\lambda), \quad \lambda=\left(\lambda_{1}, \lambda_{2}, \ldots, \lambda_{N}\right) .
$$

Let $\bar{m}_{N}$ and $m$ be the Stieltjes transforms of the density $p_{N}^{(1)}$ and the equilibrium density $\varrho$, respectively. Notice that in Section 3 we have used $m=m_{N}$ to denote the 
Stieltjes transform of the empirical measure (3.2); here $\bar{m}_{N}$ denotes the ensemble average of the analogous quantity.

Define the analytic functions

$$
b_{N}(z):=\int_{\mathbb{R}} \frac{V^{\prime}(z)-V^{\prime}(t)}{z-t}\left(p_{1}^{(N)}-\varrho\right)(t) \mathrm{d} t
$$

and $c_{N}(z):=\frac{1}{N^{2}} k_{N}(z)+\frac{1}{N}\left(\frac{2}{\beta}-1\right) \bar{m}_{N}^{\prime}(z)$, where $k_{N}(z):=\operatorname{var}_{\mu}\left(\sum_{k=1}^{N} \frac{1}{z-\lambda_{k}}\right)$. Here for complex random variables $X$ we use the definition that $\operatorname{var}(X)=\mathbb{E}\left(X^{2}\right)-$ $\mathbb{E}(X)^{2}$.

The equation used by Johansson (which can be obtained by a change of variables in (6.4), 47], or by integration by parts [61]), is a variation of the loop equation (see, e.g., 41]) used in the physics literature and it takes the form

$$
\left(\bar{m}_{N}-m\right)^{2}+s\left(\bar{m}_{N}-m\right)+b_{N}=c_{N} .
$$

Equation (6.5) expresses the difference $\bar{m}_{N}-m$ in terms of $\left(\bar{m}_{N}-m\right)^{2}, b_{N}$ and $c_{N}$. In the regime where $\left|\bar{m}_{N}-m\right|$ is small, we can neglect the quadratic term. The term $b_{N}$ is of the same order as $\left|\bar{m}_{N}-m\right|$ and is difficult to treat. As observed in [2, 61, for analytic $V$, this term vanishes when we perform a contour integration. So we have roughly the relation

$$
\left(\bar{m}_{N}-m\right) \sim \frac{1}{N^{2}} \operatorname{var}_{\mu}\left(\sum_{k=1}^{N} \frac{1}{z-\lambda_{k}}\right),
$$

where we dropped the less important error involving $\bar{m}_{N}^{\prime}(z) / N$ due to the extra $1 / N$ factor. In the convex setting, the variance can be estimated by the logarithmic Sobolev inequality and we immediately obtain an estimate on $\bar{m}_{N}-m$. We then use the Helffer-Sjöstrand formula, see (3.9), to estimate the locations of the particles. This will provide us with an accuracy of order $N^{-1 / 2}$ for $\mathbb{E}_{\mu} \lambda_{k}-\gamma_{k}$. This argument gives only an estimate on the expectation of the locations of the particles since we only have information on the averaged quantity, $\bar{m}_{N}$. Although it is tempting to use this new accuracy information on the particles to estimate the variance again in (6.6), the information on the expectation on $\lambda_{k}$ alone is very difficult to use in a bootstrap argument. To estimate the variance of a non-trivial function of $\lambda_{k}$, we need high probability estimates on $\lambda_{k}$.

The key idea in this section is the observation that the accuracy information on the $\lambda$ 's can be used to improve the local convexity of the measure $\mu$ in the direction involving the differences of $\lambda$ 's. To explain this idea, we compute the Hessian of the Hamiltonian of $\mu$ :

$$
\left\langle\mathbf{v}, \nabla^{2} \mathcal{H}(\lambda) \mathbf{v}\right\rangle \geq \vartheta\|\mathbf{v}\|^{2}+\frac{1}{N} \sum_{i<j} \frac{\left(v_{i}-v_{j}\right)^{2}}{\left(\lambda_{i}-\lambda_{j}\right)^{2}} .
$$

The naive lower bound on $\nabla^{2} \mathcal{H}$ is $\vartheta$, but for a typical $\lambda=\left(\lambda_{1}, \lambda_{2}, \ldots, \lambda_{N}\right)$ it is in fact much better in most directions. To see this effect, suppose we know $\left|\lambda_{i}-\lambda_{j}\right| \lesssim M / N$ with some $M$ for any $i, j \in I_{k}^{M}$, where $I_{k}^{M}:=\llbracket k-M, k+M \rrbracket$. Then for $\mathbf{v}=\left(v_{k-M}, \ldots, v_{k+M}\right)$ with $\sum_{j} v_{j}=0$, we have

$$
\left\langle\mathbf{v}, \nabla^{2} \mathcal{H}(\lambda) \mathbf{v}\right\rangle \geq \frac{N}{M^{2}} \sum_{i, j \in I_{k}^{M}}\left(v_{i}-v_{j}\right)^{2} \geq C \frac{N}{M} \sum_{j} v_{j}^{2} .
$$


This improves the convexity of the Hessian to $N / M$ on the hyperplane $\sum_{j} v_{j}=0$. Let

$$
\lambda_{k}^{[M]}:=\left|I_{k}^{M}\right|^{-1} \sum_{j \in I_{k}^{M}} \lambda_{j}
$$

denote the block average of the locations of particles, and rewrite

$$
\lambda_{k}-\lambda_{k}^{\left[N^{1-\varepsilon}\right]}=\sum_{j}\left(\lambda_{k}^{\left[M_{j}\right]}-\lambda_{k}^{\left[M_{j+1}\right]}\right)
$$

as a telescopic sum with an appropriate sequence of $M_{1}=0, M_{2}, \ldots$ We can now use the improved concentration on the hyperplane $\sum_{j} v_{j}=0$ to the variables $\lambda_{k}^{\left[M_{j}\right]}-\lambda_{k}^{\left[M_{j+1}\right]}$ to control the fluctuation of $\lambda_{k}-\lambda_{k}^{\left[N^{1-\varepsilon}\right]}$. Since the fluctuation of $\lambda_{k}^{\left[N^{1-\varepsilon}\right]}$ is very small for small $\varepsilon$, we finally arrive at the estimate

$$
\mathbb{P}_{\mu}\left(\left|\lambda_{k}-\mathbb{E}_{\mu}\left(\lambda_{k}\right)\right|>a\right) \leq C e^{-C N^{2} a^{2} / M} .
$$

From (6.8) we thus have that $\left|\lambda_{k}-\mathbb{E}_{\mu} \lambda_{k}\right| \lesssim \sqrt{M} / N$ with high probability. This improves the starting accuracy $\left|\lambda_{i}-\lambda_{j}\right| \lesssim M / N$ for $i, j \in I_{k}^{M}$ to $\left|\lambda_{i}-\lambda_{j}\right| \lesssim M^{\prime} / N$ with some $M^{\prime} \ll M$, provided we can prove that $\left|\mathbb{E}_{\mu}\left(\lambda_{i}-\lambda_{j}\right)\right| \ll M^{\prime} / N$. But the last inequality involves only expectations, and it will follow from the analysis of the loop equation (6.5) we just mentioned above. Starting from $M=N$, this procedure can be repeated by decreasing $M$ step by step until we get the optimal accuracy, $M \sim O(1)$. The implementation of this argument in [10] is somewhat different from this sketch due to various technical issues, but it follows the same basic idea.

\section{Beta ensemble: the Local EQuilibrium measure}

Having completed the first step, the rigidity estimate, we now focus on the second step, i.e., on the uniqueness of the local Gibbs measure. Let $0<\kappa<1 / 2$. Choose $q \in[\kappa, 1-\kappa]$ and set $L=[N q]$ (the integer part). Fix an integer $K=N^{k}$ with $k<1$. We will study the local spacing statistics of $K$ consecutive particles

$$
\left\{\lambda_{j}: j \in I\right\}, \quad I=I_{L}:=\llbracket L+1, L+K \rrbracket .
$$

These particles are typically located near $E_{q}$ determined by the relation

$$
\int_{-\infty}^{E_{q}} \varrho(t) \mathrm{d} t=q
$$

Note that $\left|\gamma_{L}-E_{q}\right| \leq C / N$.

We will distinguish the inside and outside particles by renaming them as

$$
\left(\lambda_{1}, \lambda_{2}, \ldots, \lambda_{N}\right):=\left(y_{1}, \ldots, y_{L}, x_{L+1}, \ldots, x_{L+K}, y_{L+K+1}, \ldots, y_{N}\right) \in \Xi^{(N)},
$$

but note that they keep their original indices. The notation $\Xi^{(N)}$ refers to the simplex $\left\{\mathbf{z}: z_{1}<z_{2}<\cdots<z_{N}\right\}$ in $\mathbb{R}^{N}$. In short we will write

$$
\mathbf{x}=\left(x_{L+1}, \ldots, x_{L+K}\right) \quad \text { and } \quad \mathbf{y}=\left(y_{1}, \ldots, y_{L}, y_{L+K+1}, \ldots, y_{N}\right),
$$

all in increasing order, i.e., $\mathbf{x} \in \Xi^{(K)}$ and $\mathbf{y} \in \Xi^{(N-K)}$. We will refer to the $y$ 's as external points and to the $x$ 's as internal points. 
We will fix the external points (also called as boundary conditions) and study conditional measures on the internal points. We define the local equilibrium measure on $\mathbf{x}$ with fixed boundary condition $\mathbf{y}$ by

$$
\mu_{\mathbf{y}}(\mathrm{d} \mathbf{x})=\mu_{\mathbf{y}}(\mathbf{x}) \mathrm{d} \mathbf{x}, \quad \mu_{\mathbf{y}}(\mathbf{x}):=\mu(\mathbf{y}, \mathbf{x})\left[\int \mu(\mathbf{y}, \mathbf{x}) \mathrm{d} \mathbf{x}\right]^{-1} .
$$

Note that for any fixed $\mathbf{y} \in \Xi^{(N-K)}$, the measure $\mu_{\mathbf{y}}$ is supported on configurations of $K$ points $\mathbf{x}=\left\{x_{j}\right\}_{j \in I}$ located in the interval $\left[y_{L}, y_{L+K+1}\right]$.

The Hamiltonian $\mathcal{H}_{\mathbf{y}}$ of the measure $\mu_{\mathbf{y}}(\mathrm{d} \mathbf{x}) \sim \exp \left(-N \mathcal{H}_{\mathbf{y}}(\mathbf{x})\right) \mathrm{d} \mathbf{x}$ is given by

$$
\begin{aligned}
\mathcal{H}_{\mathbf{y}}(\mathbf{x}):=\sum_{i \in I} \frac{\beta}{2} V_{\mathbf{y}}\left(x_{i}\right)-\frac{\beta}{N} \sum_{\substack{i, j \in I \\
i<j}} \log \left|x_{j}-x_{i}\right| \\
\text { with } \quad V_{\mathbf{y}}(x):=V(x)-\frac{1}{N} \sum_{j \notin I} \log \left|x-y_{j}\right| .
\end{aligned}
$$

We now define the set of good boundary configurations with a parameter $\delta=\delta(N)>$ 0 :

$$
\begin{aligned}
\mathcal{G}_{\delta}=\mathcal{G}:=\left\{\mathbf{y} \in \Xi^{(N-K)}:\left|y_{j}-\gamma_{j}\right| \leq \delta,\right. \\
\quad \forall j \in \llbracket N \kappa / 2, L \rrbracket \cup \llbracket L+K+1, N(1-\kappa / 2) \rrbracket\},
\end{aligned}
$$

where $\kappa$ is a small constant to cutoff points near the spectral edges. Some rather weak additional conditions for $\mathbf{y}$ near the spectral edges will also be needed, but we will neglect this issue here.

Let $\sigma$ and $\mu$ be two measures of the form (1.7) with potentials $W$ and $V$ and densities $\varrho=\varrho_{W}$ and $\varrho_{V}$, respectively. For our purposes $W(x)=x^{2} / 2$, i.e., $\sigma$ is the Gaussian $\beta$-ensemble and $\varrho_{W}(t)=\frac{1}{2 \pi}\left(4-t^{2}\right)_{+}^{1 / 2}$ is the Wigner semicircle law. Let the sequence $\gamma_{j}$ be the classical locations for $\mu$, and let the sequence $\theta_{j}$ be the classical locations for $\sigma$. Similarly to the construction of the measure $\mu_{\mathbf{y}}$, for any positive integer $L^{\prime} \in \llbracket 1, N-K \rrbracket$, we can construct the measure $\sigma_{\boldsymbol{\theta}}$ conditioned that the particles outside are given by the classical locations $\theta_{j}$ for $j \notin \llbracket L^{\prime}, L^{\prime}+K \rrbracket$. More precisely, we define a reference local Gaussian measure $\sigma_{\theta}$ on the set $\left[\theta_{L^{\prime}}, \theta_{L^{\prime}+K+1}\right]$ via the Hamiltonian

$$
\mathcal{H}_{\theta}(\mathbf{x})=\sum_{i \in I^{\prime}}\left[\frac{\beta}{4} x_{i}^{2}-\frac{\beta}{N} \sum_{j \notin I^{\prime}} \log \left|x_{i}-\theta_{j}\right|\right]-\frac{\beta}{N} \sum_{\substack{i, j \in I^{\prime} \\ i<j}} \log \left|x_{j}-x_{i}\right|,
$$

where $I^{\prime}:=\llbracket L^{\prime}+1, L^{\prime}+K \rrbracket$. Since $L^{\prime}$ will not play an active role, we will abuse the notation and set $L^{\prime}=L$.

The measure $\mu_{\mathbf{y}}$ lives on the interval $\left[y_{L}, y_{L+K+1}\right]$ while the measure $\sigma_{\boldsymbol{\theta}}$ lives on the interval $\left[\theta_{L}, \theta_{L+K+1}\right]$, and it is difficult to compare them. But after an appropriate translation and dilation, they will live on the same interval, and from now on we assume that $\left[y_{L}, y_{L+K+1}\right]=\left[\theta_{L}, \theta_{L+K+1}\right]$. The parameter $K=N^{k}$ has to be sufficiently small since $\varrho_{V}$ and $\varrho_{W}$ are not constant functions and we have to match these two densities quite precisely in the whole interval. There are some other subtle issues related to the rescaling, but we will neglect them here to concentrate on the main ideas. Our main result is the following theorem, which is essentially a combination of Proposition 4.2 and Theorem 4.4 from [10]. 
Theorem 7.1. Let $0<\varphi \leq \frac{1}{38}$. Fix $K=N^{k}, \delta=N^{-d}$ with $d=1-\varphi$ and $k=\frac{39}{2} \varphi$. Then for $\mathbf{y} \in \mathcal{G}$, we have

$$
\left|\mathbb{E}_{\mu_{\mathbf{y}}} \frac{1}{K} \sum_{i \in I} G\left(N\left(x_{i}-x_{i+1}\right)\right)-\mathbb{E}_{\sigma_{\theta}} \frac{1}{K} \sum_{i \in I} G\left(N\left(x_{i}-x_{i+1}\right)\right)\right| \rightarrow 0
$$

as $N \rightarrow \infty$ for any smooth and compactly supported test function $G$. A similar formula holds for more complicated observables of the form (2.10).

The basic idea for proving Theorem 7.1 is to use the Dirichlet form inequality (2.33). Although (2.33) was stated for an infinite volume measure, it holds for any measure with repulsive logarithmic interactions in a finite volume and with the parameter $\tau^{-1}$ being the lower bound on the Hessian of the Hamiltonian. In our setting, we denote by $\tau_{\sigma}^{-1}$ the lower bound for $\nabla^{2} \mathcal{H}_{\sigma}$, and the Dirichlet form inequality becomes

$$
\begin{aligned}
& \left|\left[\mathbb{E}_{\mu_{\mathbf{y}}}-\mathbb{E}_{\sigma_{\boldsymbol{\theta}}}\right] \frac{1}{K} \sum_{i \in I} G\left(N\left(x_{i}-x_{i+1}\right)\right)\right| \\
& \quad \leq C\left(\frac{\tau_{\sigma} N^{\varepsilon}}{K} D\left(\mu_{\mathbf{y}} \mid \sigma_{\theta}\right)\right)^{1 / 2}+C e^{-c N^{\varepsilon}} \sqrt{S\left(\mu_{\mathbf{y}} \mid \sigma_{\boldsymbol{\theta}}\right)}
\end{aligned}
$$

where

$$
D\left(\mu_{\mathbf{y}} \mid \sigma_{\boldsymbol{\theta}}\right):=\frac{1}{2 N} \int\left|\nabla \sqrt{\frac{\mathrm{d} \mu_{\mathbf{y}}}{\mathrm{d} \sigma_{\boldsymbol{\theta}}}}\right|^{2} \mathrm{~d} \sigma_{\boldsymbol{\theta}}
$$

Thus our task is to prove that

$$
\tau_{\sigma} N^{\varepsilon} \frac{D\left(\mu_{\mathbf{y}} \mid \sigma_{\boldsymbol{\theta}}\right)}{K} \rightarrow 0
$$

By definition,

$$
\frac{\tau_{\sigma}}{K} D\left(\mu_{\mathbf{y}} \mid \sigma_{\theta}\right) \leq \frac{\tau_{\sigma} N}{K} \int \sum_{L+1 \leq j \leq L+K} Z_{j}^{2} \mathrm{~d} \mu_{\mathbf{y}}
$$

where $Z_{j}$ is defined as

$$
Z_{j}:=\frac{\beta}{2} V^{\prime}\left(x_{j}\right)-\frac{\beta}{N} \sum_{\substack{k<L \\ k>L+K}} \frac{1}{x_{j}-y_{k}}-\frac{\beta}{2} W^{\prime}\left(x_{j}\right)+\frac{\beta}{N} \sum_{\substack{k<L \\ k>L+K}} \frac{1}{x_{j}-\theta_{k}} .
$$

Using the equilibrium relation (1.10) between the potentials $V, W$ and the densities $\varrho_{V}, \varrho_{W}$, we have

$$
Z_{j}=\beta \int_{\mathbb{R}} \frac{\varrho_{V}(y)}{x_{j}-y} \mathrm{~d} y-\frac{\beta}{N} \sum_{\substack{k<L \\ k>L+K}} \frac{1}{x_{j}-y_{k}}-\beta \int_{\mathbb{R}} \frac{\varrho_{W}(y)}{x_{j}-y} \mathrm{~d} y+\frac{\beta}{N} \sum_{\substack{k<L \\ k>L+K}} \frac{1}{x_{j}-\theta_{k}} .
$$

Hence $Z_{j}$ is the sum of the error terms,

$$
\begin{aligned}
A_{j} & :=\int_{y \notin\left[y_{L}, y_{L+K+1}\right]} \frac{\varrho_{V}(y)}{x_{j}-y} \mathrm{~d} y-\frac{1}{N} \sum_{\substack{k<L \\
k>L+K}} \frac{1}{x_{j}-y_{k}}, \\
B_{j} & :=\int_{y_{L}}^{y_{L+K+1}} \frac{\varrho_{V}(y)-\varrho_{W}(y)}{x_{j}-y} \mathrm{~d} y,
\end{aligned}
$$

and there is a term similar to $A_{j}$ with $y_{j}$ replaced by $\theta_{j}$ and $\varrho_{V}$ replaced by $\varrho_{W}$. 
With our convention, the total numbers of particles in the interval $\left[y_{L+K+1}, y_{L}\right]$ are equal, and thus

$$
\int_{y_{L}}^{y_{L+K+1}} \varrho_{V}(y) \mathrm{d} y=\int_{y_{L}}^{y_{L+K+1}} \varrho_{W}(y) \mathrm{d} y .
$$

Since the densities $\rho_{V}$ and $\rho_{W}$ are $C^{1}$ functions away from the endpoints $A$ and $B$ and $y_{L+K+1}-y_{L}$ is small, $\left|\rho_{V}-\rho_{W}\right|$ is small in the interval $\left[y_{L+K+1}, y_{L}\right]$ and thus $B_{j}$ is small. For estimating $A_{j}$, we can replace the integral $\int_{-\infty}^{y_{L}} \frac{\varrho_{V}(y)}{x_{j}-y} \mathrm{~d} y$ by $\frac{1}{N} \sum_{k<L} \frac{1}{x_{j}-\gamma_{k}}$ with negligible errors, at least for $j$ 's away from the edges, $j \in$ $\llbracket L+N^{\varepsilon}, L+K-N^{\varepsilon} \rrbracket$. Thus

$$
\left|A_{j}\right| \leq \frac{C}{N}\left|\sum_{\substack{k<L \\ k>L+K}} T_{j}^{k}\right|, \quad T_{j}^{k}:=\frac{1}{x_{j}-y_{k}}-\frac{1}{x_{j}-\gamma_{k}},
$$

and $T_{j}^{k}$ can be estimated by the assumption $\left|y_{k}-\gamma_{k}\right| \leq \delta$ from $\mathbf{y} \in \mathcal{G}$. The same argument works if $j$ is close to the edge, but $k$ is away from the edges, i.e., $k \leq L-N^{\varepsilon}$ or $k \geq L+K+N^{\varepsilon}$. The edge terms, $T_{j}^{k}$ for $|j-k| \leq N^{\varepsilon}$, are difficult to estimate due to the singularity in the denominator and the event that many $y_{k}$ 's with $k<L$ may pile up near $y_{L}$. To resolve this difficulty, we show that the averaged local statistics of the measure $\mu_{\mathbf{y}}$ are insensitive to the change of the boundary conditions for $\mathbf{y}$ near the edges. This can be achieved by the simple inequality

$$
\left|\frac{1}{K} \sum_{i \in I} \int G\left(N\left(x_{i}-x_{i+1}\right)\right)\left[\mathrm{d} \mu_{\mathbf{y}^{\prime}}-\mathrm{d} \mu_{\mathbf{y}}\right]\right| \leq C \int\left|\mathrm{d} \mu_{\mathbf{y}^{\prime}}-\mathrm{d} \mu_{\mathbf{y}}\right| \leq C \sqrt{S\left(\mu_{\mathbf{y}^{\prime}} \mid \mu_{\mathbf{y}}\right)}
$$

for any two boundary conditions $\mathbf{y}$ and $\mathbf{y}^{\prime}$. Although we still have to estimate the entropy that includes a logarithmic singularity, this can be done much more easily. Therefore, we can replace the boundary condition $y_{k}$ with $y_{k}^{\prime}=\theta_{k}$ for $|j-k| \leq N^{\varepsilon}$, and then the most singular edge terms in (7.10) cancel out.

We note that we can perform this replacement only for a small number of index pairs $(j, k)$, since estimating the gap distribution by the total entropy, as noted in (2.36) in Section 2, is not as efficient as the estimate using the Dirichlet form per particle. Thus we can afford to use this argument only for the edge terms, $|j-k| \leq N^{\varepsilon}$. For all other index pairs $(j, k)$ we still have to estimate $T_{j}^{k}$ by exploiting that $\mathbf{y}$ is a good configuration, i.e., $y_{k}-\gamma_{k}$ is small.

Unfortunately, even with the optimal accuracy $\delta \sim N^{-1+\varepsilon^{\prime}}$ in (7.4) as an input, the relation (7.9) still cannot be satisfied for any choice of $N^{c \varepsilon^{\prime}} \leq K \leq N^{1-c \varepsilon^{\prime}}$. We do not know whether this is due to our handling of the edge terms or some other intrinsic reasons. To understand why this might occur, we remark that while the edge terms become a smaller percentage of the total terms in (7.14) as $K$ gets bigger, the relaxation time to equilibrium for $\sigma_{\boldsymbol{\theta}}$, determined by the convexity of $\mathcal{H}_{\boldsymbol{\theta}}^{\prime \prime}$, increases at the same time. At the end of our calculation, there is no good regime for the choice of $K$. Fortunately, this can be resolved by using the idea of the local relaxation measure [34, i.e., we add a quadratic term $\frac{1}{2 \tau}\left(x_{j}-\gamma_{j}\right)^{2}$ to the measure $\mu_{\mathbf{y}}$ and $\frac{1}{2 \tau_{\sigma}}\left(x_{j}-\theta_{j}\right)^{2}$ to the measure $\sigma_{\boldsymbol{\theta}}$. With these ideas, we can complete the proof of Theorem 7.1 . 


\section{More general Classes of RAndom matrices}

All our results concerning Wigner matrices hold for a broader class of ensembles where the matrix elements $h_{i j}$ still have mean zero, $\mathbb{E} h_{i j}=0$, but their variances are allowed to vary. More precisely, we assume that the variances $\sigma_{i j}^{2}:=\mathbb{E}\left|h_{i j}\right|^{2}$ satisfy the normalization condition

$$
\sum_{j=1}^{N} \sigma_{i j}^{2}=1, \quad i=1,2, \ldots, N
$$

and they are comparable, i.e.,

$$
0<C_{\mathrm{inf}} \leq N \sigma_{i j}^{2} \leq C_{\text {sup }}<\infty, \quad i, j=1,2, \ldots, N,
$$

for some fixed positive constants $C_{\text {inf }}$ and $C_{\text {sup }}$. These ensembles are called generalized Wigner ensembles. In the special case $\sigma_{i j}^{2}=1 / N$, we recover the original Wigner ensemble. All our results concerning the bulk universality, delocalization of eigenvectors, and local semicircle laws hold for generalized Wigner matrices as well.

There is another important class of random matrices, the band matrices, which are characterized by the property that $\sigma_{i j}^{2}$ is a function of $|i-j|$ on scale $W$, which is called the bandwidth, i.e.,

$$
\sigma_{i j}^{2}=W^{-1} f\left(\frac{[i-j]_{N}}{W}\right)
$$

where $f: \mathbb{R} \rightarrow \mathbb{R}_{+}$is a bounded non-negative symmetric function with $\int f=1$ and $[i-j]_{N} \equiv i-j \bmod N$. For this class, the local semicircle law is known to hold at least down to scale $\eta \sim W^{-1}$ and all eigenvectors are delocalized at least on scale $W$. Moreover, most eigenvectors are known to be delocalized on a much larger scale $W^{7 / 6}$ [23, 24], but smaller than $W^{8}$ [58, and it is expected that the correct localization length is $W^{2}$. So far no bulk universality result is known.

The significance of the random band matrices stems from the fact that they interpolate between discrete random Schrödinger operators with short range hoppings (Anderson model) and the Wigner matrices. In particular, random matrix spectral statistics are expected to hold in the presumed delocalization regime of the Anderson model in three or higher dimensions. For more details on this exciting connection, see 66.

Finally we mention the ensemble of sample covariance matrices that play a fundamental role in statistics. These are matrices of the form $H=A^{*} A$, where $A$ is an $M \times N$ matrix with independent identically distributed entries. The semicircle law is replaced with the Marchenko-Pastur law, but most results listed in this review remain valid. For more details, see [34, 69].

\section{EdGe Universality}

Denote by $\lambda_{N}$ is the largest eigenvalue of a generalized Wigner matrix. The probability distribution functions of $\lambda_{N}$ for the classical Gaussian ensembles are identified by Tracy and Widom [72, 73] to be

$$
\lim _{N \rightarrow \infty} \mathbb{P}\left(N^{2 / 3}\left(\lambda_{N}-2\right) \leq s\right)=F_{\beta}(s),
$$


where the functions $F_{\beta}(s)$ can be computed in terms of Painlevé equations and $\beta=1,2,4$ corresponds to the standard classical ensembles. The distribution of $\lambda_{N}$ is believed to be universal and independent of the Gaussian structure.

The local semicircle law, Theorem 3.1] combined with a modification of the Green function comparison theorem, Theorem 4.1 implies the following version of universality of the extreme eigenvalues. Although it holds for correlation functions of finite number of eigenvalues, for simplicity we state it for the largest one and for the case of symmetric matrices only.

Theorem 9.1 (Universality of the largest eigenvalue [38, Theorem 2.4]). Suppose that we have two $N \times N$ symmetric generalized Wigner matrices, $H^{(v)}$ and $H^{(w)}$, with matrix elements $h_{i j}$ given by the random variables $N^{-1 / 2} v_{i j}$ and $N^{-1 / 2} w_{i j}$, respectively, with $v_{i j}$ and $w_{i j}$ satisfying the uniform subexponential decay condition (3.3). Let $\mathbb{P}^{\mathbf{v}}$ and $\mathbb{P}^{\mathbf{w}}$ denote the probability, and let $\mathbb{E}^{\mathbf{v}}$ and $\mathbb{E}^{\mathbf{w}}$ denote the expectation with respect to these collections of random variables. Suppose that

$$
\mathbb{E}^{\mathbf{v}} v_{i j}^{2}=\mathbb{E}^{\mathbf{w}} w_{i j}^{2}
$$

Then there is an $\varepsilon>0$ depending on $\vartheta$ in (3.3) such that for any real parameter $s$ (may depend on $N$ ) we have

$$
\begin{aligned}
\mathbb{P}^{\mathbf{v}}\left(N^{2 / 3}\right. & \left.\left(\lambda_{N}-2\right) \leq s-N^{-\varepsilon}\right)-N^{-\varepsilon} \\
& \leq \mathbb{P}^{\mathbf{w}}\left(N^{2 / 3}\left(\lambda_{N}-2\right) \leq s\right) \leq \mathbb{P}^{\mathbf{v}}\left(N^{2 / 3}\left(\lambda_{N}-2\right) \leq s+N^{-\varepsilon}\right)+N^{-\varepsilon}
\end{aligned}
$$

for $N \geq N_{0}$ sufficiently large, where $N_{0}$ is independent of $s$.

Note that although Theorem 9.1 states that the edge distribution is universal for a fixed choice of the variances $\sigma_{i j}^{2}$, it does not identify this distribution. In particular, we do not know if it coincides with the Tracy-Widom distribution apart from the Hermitian case, when the method of [47] can be applied. The extension of Theorem 9.1 to eigenvectors was recently obtained by Knowles and Yin [48, i.e., under the assumption (9.2), the distributions for the largest eigenvectors coincide. Similar results hold for the joint distribution of eigenvectors near the edges.

\section{ERDŐS-RÉNYI MATRIX}

The Erdös-Rényi matrix is the adjacency matrix of the Erdős-Rényi random graph [39, 40]. Its entries are independent (up to the constraint that the matrix be symmetric) and are equal to 1 with probability $p$ and 0 with probability $1-p$. We rescale the matrix in such a way that its bulk eigenvalues typically lie in an interval of size of order one. Thus we have a symmetric $N \times N$ matrix $A=\left(a_{i j}\right)$ whose entries $a_{i j}$ are independent (up to the symmetry constraint $a_{i j}=a_{j i}$ ) and each element is distributed according to

$$
a_{i j}=\frac{\gamma}{q}\left\{\begin{array}{ll}
1 & \text { with probability } \frac{q^{2}}{N}, \\
0 & \text { with probability } 1-\frac{q^{2}}{N},
\end{array}:=\sqrt{p N} .\right.
$$

Here $\gamma:=\left(1-q^{2} / N\right)^{-1 / 2}$ is a scaling introduced for convenience to compare with Wigner matrices. We also assume that $q=\sqrt{p N} \geq(\log N)^{C \log \log N}$, in particular the Erdős-Rényi graph is connected.

Theorem 10.1 (Local semicircle law for Erdős-Rényi matrix [25, Theorem 2.9]). Let $m(z)$ denote the Stieltjes transform of the empirical eigenvalue distribution of the matrix $A$, and let $G(z)=(A-z)^{-1}$ be its resolvent. Assume that the spectral 
parameter $z=E+$ in satisfies $|E| \leq 5$ and $(\log N)^{L} N^{-1} \leq \eta \leq 3$ with a sufficiently large constant $L$. Then we have the following two estimates:

(i) The Stieltjes transform of the empirical eigenvalue distribution of A satisfies

$$
\mathbb{P}\left\{\left|m(z)-m_{s c}(z)\right| \geq(\log N)^{C}\left[\frac{1}{N \eta}+\frac{1}{q}\right]\right\} \leq C \exp \left[-c(\log N)^{c}\right] .
$$

(ii) The individual matrix elements of the Green function satisfy that

$$
\begin{aligned}
& \mathbb{P}\left\{\max _{i, j}\left|G_{i j}(z)-\delta_{i j} m_{s c}(z)\right| \geq(\log N)^{C}\left[\sqrt{\frac{\mathfrak{I m} m_{s c}(z)}{N \eta}}+\frac{1}{N \eta}+\frac{1}{q}\right]\right\} \\
& \leq C \exp \left[-c(\log N)^{c}\right] .
\end{aligned}
$$

Compared with the local semicircle law, Theorem 3.1. there is an extra factor $1 / q$ appearing in the error estimates of Theorem 10.1. This extra error term affects the rigidity estimate of eigenvalues, and (3.2) becomes

$$
\left|\mu_{j}-\gamma_{j}\right| \leq(\log N)^{C}\left[N^{-2 / 3} j^{-1 / 3}+q^{-2}\right], \quad j \leq N / 2,
$$

for $q=\sqrt{p N} \gg N^{1 / 3}$. We also have an estimate for the regime $q \leq N^{1 / 3}$, but that is weaker. Moreover, under the assumption $q \gg N^{1 / 3}$, both bulk and edge universality are proved (see Theorem 2.5 and 2.7 in [25]). It is well known that the largest eigenvalue $\lambda_{N}$ of $A$ satisfies

$$
\lambda_{N}=\gamma q+\frac{1}{\gamma q}+o(1)
$$

hence it is located far away from the bulk spectrum. Therefore, the edge universality for Erdős-Rényi matrices refers to the second largest eigenvalue instead of the largest one. Since the matrix elements of $A$ have non-zero means, both the edge and bulk universality require substantial new ideas in addition to those we have sketched. We refer the interested readers to the original papers [25, 26] for more detailed explanations.

\section{Historical REMARKS}

Finally, we summarize the recent history related to the universality of local eigenvalue statistics of Wigner matrices. The three-step approach was first introduced in [27] in the context of Hermitian Wigner matrices, and it led to the first proof of the Wigner-Dyson-Gaudin-Mehta conjecture for Hermitian Wigner matrices. It works whenever the distributions of the matrix elements are smooth. This approach was followed by all later works on the bulk universalities. We now review the history of Steps 1-3 separately, and we start with the history of Step 1, the local semicircle law.

The semicircle law was proved by Wigner for energy windows of order one. Various improvements were made to shrink the spectral windows; in particular, results down to scale $N^{-1 / 2}$ were obtained by 4 and 44 . The result at the optimal scale, $N^{-1}$, referred to as the local semicircle law, was established for Wigner matrices in a series of papers [30, 31, 32. The method was based on a self-consistent equation for the Stieltjes transform of the eigenvalues, $m(z)$, and the continuity in the imaginary part of the spectral parameter $z$. As a byproduct, the optimal eigenvector delocalization estimate was proved. In order to deal with the generalized Wigner matrices, we needed to consider the self-consistent equation of 
$G_{i j}(z)$, the matrix elements of the Green function, since there is no closed equation for $m(z)=N^{-1} \operatorname{Tr} G(z)$ [36, 37]. In particular, this method implied the optimal rigidity estimate of eigenvalues in the bulk in [37] and up to the edges in [38]. The estimate on $G_{i i}$ provided a simple alternative proof of the eigenvector delocalization estimate. The extension of the local semicircle law to the Erdős-Rényi matrices was recently made in [25].

We now review the history of Step 2. Recall that Hermitian Gaussian divisible ensembles are matrices of the form $e^{-t / 2} H_{0}+\left(1-e^{-t}\right)^{1 / 2} U$, where $U$ is the GUE and $H_{0}$ is a Wigner ensemble. The universality of this ensemble for a large class of $H_{0}$ and for parameters $t$ of order one was proved by Johansson [46]. It was extended to complex sample covariance matrices by Ben Arous and Péché [6]. There were two major restrictions of this method:

1. The Gaussian component was fairly large and it was required to be of order one independent of $N$.

2. The method relies on an explicit formula by Brézin and Hikami 12 for the correlation functions of eigenvalues.

This formula originates in the Harish-Chandra/Itzykson/Zuber integral [45] and it is valid only for Gaussian divisible ensembles with unitary invariant Gaussian component. The size of the Gaussian component was reduced to $N^{-1+\varepsilon}$ in [27] by using an improved formula for correlation functions and the local semicircle law from 30, 31, 32.

To eliminate the usage of an explicit formula, a conceptual approach for Step 2 via the local ergodicity of Dyson Brownian motion was initiated in 33 . In this paper, the first version of the local relaxation flow was introduced, but it was rather complicated. In [34] we found a much simpler way to enhance the convexity of the Dyson Brownian motion, and we proved a general theorem for local ergodicity of DBM and related flow, i.e., Theorem 2.1. This theorem applies to all classical ensembles, i.e., real and complex Wigner matrices, real and complex sample covariance matrices, and quaternion Wigner matrices. The local relaxation flow in the simple form (2.25) first appeared in 34. The relaxation time to local equilibrium proved in these two papers was not optimal; the optimal relaxation time, conjectured by Dyson, was obtained later in [38.

The third and final step is to approximate the local eigenvalue distribution of a general Wigner matrix by that of a Gaussian divisible one. The first approximation result was obtained via the reversal heat flow in [27] which required some smoothness of the distribution of matrix elements. Shortly after, Tao and Vu [67, proved a comparison theorem with a four moment matching condition. Instead of using a Gaussian divisible ensemble with a small $\left(N^{-1+\varepsilon}\right)$ Gaussian component, they relied on Johansson's result [46 to provide Hermitian Gaussian divisible ensembles for comparison. This proved the universality of Hermitian Wigner matrices, provided that the distributions of matrix elements have vanishing third moment and are supported on at least three points. These conditions were removed in 28 by combining the arguments of [27] and [67].

Due to the lack of a Brézin-Hikami type formula for the symmetric matrices, there was no extension of Johansson's result [46] to this case, and the universality for symmetric Wigner ensembles was much more difficult to prove. However, the result of 67 implies that the local eigenvalue statistics of symmetric Wigner matrices and GOE are the same, but under the restriction that the first four moments of 
the matrix elements exactly match those of GOE. The resolution of the WignerDyson-Gaudin-Mehta conjecture for symmetric matrices, i.e., Theorem 2.1 for real symmetric matrices, was obtained in [33, 36]. In these papers, two new ideas were introduced: the local relaxation flow 33 and the Green function comparison theorem [36]. Starting from the paper [36], the variances were allowed to vary and the universality was extended to generalized Wigner matrices. The real Bernoulli random matrices required a more refined argument [37. Finally, the technical condition assumed in all these papers, i.e., that the probability distributions of the matrix elements decay subexponentially, was reduced to the $(4+\varepsilon)$-moment assumption (1.8) by using the universality of Erdős-Rényi matrices [26].

The Green function comparison theorem, Theorem 4.1 uses the same four moment conditions which appeared earlier in 67, but it compares matrix elements of Green functions at a fixed energy and not just traces of Green functions which carry information on eigenvalues near a fixed energy. The result of [67], on the other hand, concerns individual eigenvalues with fixed labels. Both proofs used the local semicircle law and Lindeberg's idea (introduced in his proof of the central limit theorem). Lindeberg's idea in the context of random matrices appeared earlier in a proof of the Wigner semicircle law by Chatterjee [13. The approach 67] requires additional difficult estimates due to singularities from neighboring eigenvalues, but the Green function comparison theorem follows directly from the local semicircle law in Step 1, i.e., Theorem 3.1, via standard resolvent expansions. The difficulties associated with the singularities of eigenvalue resonances are completely absent in the Green function comparison theorem. Finally, we mention that Green function comparison can also yield comparison of eigenvalues with fixed labels; see the recent work by Knowles and Yin 48.

The edge universality for Wigner matrices was first proved via the moment method by Soshnikov 65] (see also the earlier work 62]) for Hermitian and symmetric ensembles with symmetric distributions. By combining the moment method and Chebyshev polynomials, Sodin [63, 64, proved edge universality of certain band matrices and some special class of sparse matrices with symmetric distribution. The symmetry assumption was partially removed in [59, 60]. The edge universality without any symmetry assumption was proved in 68] under the condition that the distribution of matrix elements is subexponential decay and the first three moments match those of a Gaussian distribution. The subexponential decay condition is not optimal for edge universality, in fact the finiteness of the fourth moment was conjectured to be sufficient. For Gaussian divisible Hermitian ensembles this was proved in [47. This is optimal, since on the other hand, the result by Auffinger, Ben Arous, and Péché [3] showed that the distribution of the largest eigenvalues converges to a Poisson process if the entries have at most $4-\varepsilon$ moments. For Wigner matrices with arbitrary symmetry class, the edge universality was proved under the sole assumption that the matrix entries have $12+\varepsilon$ moments [26]. Finally, we mention that extension of universality to eigenvectors near the edge was obtained by Knowles and Yin [48] under the two moment matching condition and with four moment matching condition in 70].

Although we have focused only on Wigner matrices and $\beta$-ensembles, the ideas summarized in this review should be applicable to a wide class of matrix ensembles. We have already mentioned some natural open questions related to possible 
improvements of our results. These concern removing some technical conditions such as

(i) the restriction $q \gg N^{1 / 3}$ in the bulk universality of the Erdös-Rényi matrix;

(ii) the $12+\varepsilon$ moment condition for edge universality.

A more ambitious goal would be to prove universality for systems with some spatial structure, such as band matrices or related models that may open up a path towards universality for random Schrödinger operators and other realistic models of quantum chaos.

\section{ACKNOWLEDGMENTS}

The results in this review were obtained in collaboration with Benjamin Schlein, Jun Yin, Antti Knowles, and Paul Bourgade and in some work, also with Jose Ramírez and Sandrine Péché. This article is to report the joint progress with these authors.

\section{About the Authors}

László Erdős is a professor at the Mathematical Institute of the Ludwig-Maximilians University in Munich, Germany.

Horng-Tzer Yau is a professor in the mathemematics department at Harvard University. He was awarded a MacArthur Fellowship in 2000.

\section{REFERENCES}

[1] Anderson, G., Guionnet, A., Zeitouni, O.: An Introduction to Random Matrices. Studies in advanced mathematics, 118, Cambridge University Press, 2009. MR2760897 (2011m:60016)

[2] Albeverio, S., Pastur, L., Shcherbina, M.: On the $1 / n$ expansion for some unitary invariant ensembles of random matrices, Commun. Math. Phys. 224, 271-305 (2001). MR.1869000 (2003b:82031)

[3] Auffinger, A., Ben Arous, G., Péché, S.: Poisson Convergence for the largest eigenvalues of heavy-tailed matrices. Ann. Inst. Henri Poincaré Probab. Stat. 45 (2009), no. 3, 589-610. MR.2548495 (2011b:60021)

[4] Bai, Z. D., Miao, B., Tsay, J.: Convergence rates of the spectral distributions of large Wigner matrices. Int. Math. J. 1 (2002), no. 1, 65-90. MR.1825933 (2003d:60071)

[5] Bakry, D., Émery, M.: Diffusions hypercontractives. In: Séminaire de probabilités, XIX, 1983/84, vol. 1123 of Lecture Notes in Math. Springer, Berlin, 1985, pp. 177-206. MR889476 (88j:60131)

[6] Ben Arous, G., Péché, S.: Universality of local eigenvalue statistics for some sample covariance matrices. Comm. Pure Appl. Math. LVIII. (2005), 1-42. MR2162782 (2006h:62072)

[7] Berry, M.V., Tabor, M.: Level clustering in the regular spectrum, Proc. Roy. Soc. A 356 (1977) 375-394.

[8] Bleher, P., Its, A.: Semiclassical asymptotics of orthogonal polynomials, Riemann-Hilbert problem, and universality in the matrix model. Ann. of Math. 150 (1999), 185-266. MR.1715324 (2000k:42033)

[9] Bohigas, O.; Giannoni, M.-J.; Schmit, C.: Characterization of chaotic quantum spectra and universality of level fluctuation laws. Phys. Rev. Lett. 52 (1984), no. 1, 1-4. MR730191 (85f:58034)

[10] Bourgade, P., Erdős, Yau, H.-T.: Universality of General $\beta$-Ensembles, arXiv:1104.2272

[11] Bourgade, P., Erdős, Yau, H.-T.: Bulk Universality of General $\beta$-Ensembles with Non-convex Potential, arxiv:1201.2283

[12] Brézin, E., Hikami, S.: Correlations of nearby levels induced by a random potential. Nucl. Phys. B 479 (1996), 697-706; and Spectral form factor in a random matrix theory. Phys. Rev. E 55 (1997), 4067-4083. MR1418841 (97j:82080)

[13] Chatterjee, S.: A generalization of the Lindeberg principle. Ann. Probab. 34 (2006), no. 6, 2061-2076. MR2294976(2008c:60028) 
[14] Deift, P.: Orthogonal polynomials and random matrices: a Riemann-Hilbert approach. Courant Lecture Notes in Mathematics 3, American Mathematical Society, Providence, RI, 1999 MR.1677884 (2000g:47048)

[15] Deift, P., Gioev, D.: Universality in random matrix theory for orthogonal and symplectic ensembles. Int. Math. Res. Pap. IMRP 2007, no. 2, Art. ID rpm004, 116 pp MR2335245 (2008e:82026)

[16] Deift, P., Gioev, D.: Random Matrix Theory: Invariant Ensembles and Universality. Courant Lecture Notes in Mathematics 18, American Mathematical Society, Providence, RI, 2009 MR2514781 (2011f:60008)

[17] Deift, P., Kriecherbauer, T., McLaughlin, K.T-R, Venakides, S., Zhou, X.: Uniform asymptotics for polynomials orthogonal with respect to varying exponential weights and applications to universality questions in random matrix theory. Comm. Pure Appl. Math. 52 (1999):13351425. MR1702716 (2001g:42050)

[18] Deift, P., Kriecherbauer, T., McLaughlin, K.T-R, Venakides, S., Zhou, X.: Strong asymptotics of orthogonal polynomials with respect to exponential weights. Comm. Pure Appl. Math. 52 (1999): 1491-1552. MR1711036 (2001f:42037)

[19] Dumitriu, I., Edelman, A.: Matrix Models for Beta Ensembles, Journal of Mathematical Physics 43 (11) (2002), 5830-5847. MR1936554 (2004g:82044)

[20] Dyson, F.J.: Statistical theory of energy levels of complex systems, I, II, and III. J. Math. Phys. 3, 140-156, 157-165, 166-175 (1962). MR0143556 (26:1111)

[21] Dyson, F.J.: A Brownian-motion model for the eigenvalues of a random matrix. J. Math. Phys. 3, 1191-1198 (1962). MR0148397(26:5904)

[22] Dyson, F.J.: Correlations between eigenvalues of a random matrix. Commun. Math. Phys. 19, 235-250 (1970). MR0278668(43:4398)

[23] Erdős, L., Knowles, A.: Quantum Diffusion and Eigenfunction Delocalization in a Random Band Matrix Model. Preprint. Arxiv:1002.1695

[24] Erdős, L., A. Knowles, A.: Quantum Diffusion and Delocalization for Band Matrices with General Distribution. Annales Inst. H. Poincare, 12 (7), 1227-1319 (2011).

[25] Erdős, L., Knowles, A., Yau, H.-T., Yin, J.: Spectral Statistics of Erdős-Rényi Graphs I: Local Semicircle Law. Preprint. Arxiv:1103.1919

[26] Erdős, L., Knowles, A., Yau, H.-T., Yin, J.: Spectral Statistics of Erdős-Rényi Graphs II: Eigenvalue Spacing and the Extreme Eigenvalues. Preprint. Arxiv:1103.3869

[27] Erdős, L., Péché, G., Ramírez, J., Schlein, B., and Yau, H.-T., Bulk universality for Wigner matrices. Commun. Pure Appl. Math. 63, No. 7, 895-925 (2010) MR2662426 (2011c:60022)

[28] Erdős, L., Ramirez, J., Schlein, B., Tao, T., Vu, V., Yau, H.-T.: Bulk Universality for Wigner Hermitian matrices with subexponential decay. Math. Res. Lett. 17 (2010), no. 4, 667-674. MR2661171 (2011j:60018)

[29] Erdős, L., Ramirez, J., Schlein, B., Yau, H.-T.: Universality of sine-kernel for Wigner matrices with a small Gaussian perturbation. Electr. J. Prob. 15, Paper 18, 526-604 (2010) MR2639734(2011h:60015)

[30] Erdős, L., Schlein, B., Yau, H.-T.: Semicircle law on short scales and delocalization of eigenvectors for Wigner random matrices. Ann. Probab. 37, No. 3, 815-852 (2009) MR2537522 (2010g:15036)

[31] Erdős, L., Schlein, B., Yau, H.-T.: Local semicircle law and complete delocalization for Wigner random matrices. Commun. Math. Phys. 287, 641-655 (2009) MR 2481753 (2010f:60018)

[32] Erdős, L., Schlein, B., Yau, H.-T.: Wegner estimate and level repulsion for Wigner random matrices. Int. Math. Res. Notices. 2010, No. 3, 436-479 (2010) MR2587574 (2011h:60016)

[33] Erdős, L., Schlein, B., Yau, H.-T.: Universality of random matrices and local relaxation flow. Invent. Math. 185 (2011), no.1, 75-119. MR2810797

[34] Erdős, L., Schlein, B., Yau, H.-T., Yin, J.: The local relaxation flow approach to universality of the local statistics for random matrices. Preprint arXiv:0911.3687

[35] Erdős, L., Yau, H.-T.: A comment on the Wigner-Dyson-Mehta bulk universality conjecture for Wigner matrices. Preprint arXiv:1201.5619

[36] Erdős, L., Yau, H.-T., Yin, J.: Bulk universality for generalized Wigner matrices. Preprint arXiv:1001.3453

[37] Erdős, L., Yau, H.-T., Yin, J.: Universality for generalized Wigner matrices with Bernoulli distribution. J. of Combinatorics, 1 (2011), no. 2, 15-85 MR2847916 
[38] Erdős, L., Yau, H.-T., Yin, J.: Rigidity of Eigenvalues of Generalized Wigner Matrices, preprint arXiv:1007.4652

[39] Erdős, P., Rényi, A.: On Random Graphs. I. Publicationes Mathematicae 6, $290-297$ (1959). MR0120167(22:10924)

[40] Erdős, P., Rényi, A.: The Evolution of Random Graphs. Magyar Tud. Akad. Mat. Kutató Int. Közl. 5 17-61 (1960). MR0125031(23:A2338)

[41] Eynard, B.: Master loop equations, free energy and correlations for the chain of matrices. $J$. High Energy Phys. 11 2003, 018 MR2038684(2005k:82041)

[42] Fokas, A. S., Its, A. R., Kitaev, A. V.: The isomonodromy approach to matrix models in 2D quantum gravity. Comm. Math. Phys. 147 (1992), 395-430. MR1174420(93h:81115)

[43] Gaudin, M.: Sur la loi limit de l'espacement des valeurs propres d'une matrice aléatoire. Nucl. Phys. 25, 447-458.

[44] Guionnet, A., Zeitouni, O.: Concentration of the spectral measure for large matrices. Electronic Comm. in Probability 5 (2000) Paper 14. MR1781846 (2001k:15035)

[45] Itzykson, C., Zuber, J.B.: The planar approximation, II. J. Math. Phys. 21 411-421 (1980) MR.562985 (81a:81068)

[46] Johansson, K.: Universality of the local spacing distribution in certain ensembles of Hermitian Wigner matrices. Comm. Math. Phys. 215 (2001), no.3. 683-705. MR1810949 (2002j:15024)

[47] Johansson, K.: Universality for certain Hermitian Wigner matrices under weak moment conditions. Preprint arxiv.org/abs/0910.4467

[48] Knowles, A., Yin, J.: Eigenvector distribution of Wigner matrices. Preprint arXiv:1102.0057.

[49] Kriecherbauer, T., Shcherbina, M.: Fluctuations of eigenvalues of matrix models and their applications. Preprint arXiv:1003.6121

[50] Lubinsky, D.S.: A New Approach to Universality Limits Involving Orthogonal Polynomials, Annals of Mathematics, 170, 915-939 (2009). MR2552113(2011a:42042)

[51] Mehta, M.L.: Random Matrices. Third Edition, Academic Press, New York, 1991. MR.1083764 (92f:82002)

[52] Mehta, M.L.: A note on correlations between eigenvalues of a random matrix. Commun. Math. Phys. 20 no.3. 245-250 (1971) MR0277221(43:2958)

[53] Mehta, M.L., Gaudin, M.: On the density of eigenvalues of a random matrix. Nuclear Phys. 18, 420-427 (1960). MR0112895 (22:3741)

[54] Montgomery, H.L.: The pair correlation of zeros of the zeta function. Analytic number theory, Proc. of Sympos. in Pure Math. 24, Amer. Math. Soc. Providence, R.I., 181-193 (1973). MR0337821 (49:2590)

[55] Pastur, L., Shcherbina, M.: Universality of the local eigenvalue statistics for a class of unitary invariant random matrix ensembles. J. Stat. Phys. 86, 109-147 (1997) MR.1435193 (98b:82037)

[56] Pastur, L., Shcherbina M.: Bulk universality and related properties of Hermitian matrix models. J. Stat. Phys. 130 (2008), no.2., 205-250. MR2375744 (2008k:82050)

[57] Ramirez, J., Rider, B., Virág, B.: Beta ensembles, stochastic Airy spectrum, and a diffusion. arXiv:math/0607331. To appear in J. Amer. Math. Soc.

[58] Schenker, J.: Eigenvector localization for random band matrices with power law band width. Commun. Math. Phys. 290, 1065-1097 (2009). MR2525652(2010i:60024)

[59] Péché, S., Soshnikov, A.: On the lower bound of the spectral norm of symmetric random matrices with independent entries. Electron. Commun. Probab. 13 (2008), 280-290. MR2415136 (2010c:60028)

[60] Péché, S., Soshnikov, A.: Wigner random matrices with non-symmetrically distributed entries. J. Stat. Phys. 129 (2007), no. 5-6, 857-884. MR2363385 (2008m:82046)

[61] Shcherbina, M.: Orthogonal and symplectic matrix models: universality and other properties. Preprint arXiv: 1004.2765

[62] Sinai, Y. and Soshnikov, A.: A refinement of Wigner's semicircle law in a neighborhood of the spectrum edge. Functional Anal. and Appl. 32 (1998), no. 2, 114-131. MR1647832 (2000c:82041)

[63] Sodin, S.: The spectral edge of some random band matrices. Preprint. arXiv: 0906.4047

[64] Sodin, S.: The Tracy-Widom law for some sparse random matrices. Preprint. arXiv:0903.4295

[65] Soshnikov, A.: Universality at the edge of the spectrum in Wigner random matrices. Comm. Math. Phys. 207 (1999), no.3. 697-733. MR1727234 (2001i:82037)

[66] Spencer, T.: Review article on random band matrices. Draft in preparation. 
[67] Tao, T. and Vu, V.: Random matrices: Universality of the local eigenvalue statistics, Acta Math., 206 (2011), no. 1, 127-204. MR2784665

[68] Tao, T. and Vu, V.: Random matrices: Universality of local eigenvalue statistics up to the edge. Commun. Math. Phys., 298 (2010), no. 2, 549-572. MR2669449(2011f:60012)

[69] Tao, T. and Vu, V.: Random covariance matrices: Universality of local statistics of eigenvalues. Preprint. arXiv:0912.0966

[70] Tao, T. and Vu, V.: Random matrices: Universal properties of eigenvectors. Preprint. arXiv:1103.2801

[71] Tao, T. and Vu, V.: The Wigner-Dyson-Mehta bulk universality conjecture for Wigner matrices. Preprint. arXiv:1101.5707

[72] Tracy, C., Widom, H.: Level-Spacing Distributions and the Airy Kernel, Comm. Math. Phys. 159 (1994), 151-174. MR.1257246 (95e:82003)

[73] Tracy, C., Widom, H.: On orthogonal and symplectic matrix ensembles, Comm. Math. Phys. 177 (1996), no. 3, 727-754. MR.1385083 (97a:82055)

[74] Valkó, B.; Virág, B.: Continuum limits of random matrices and the Brownian carousel. Invent. Math. 177 (2009), no. 3, 463-508. MR2534097 (2011d:60023)

[75] Widom H.: On the relation between orthogonal, symplectic and unitary matrix ensembles. J. Statist. Phys. 94 (1999), no. 3-4, 347-363. MR1675356 (2000e:82024)

[76] Wigner, E.: Characteristic vectors of bordered matrices with infinite dimensions. Ann. of Math. 62 (1955), 548-564. MR.0077805(17:1097c)

[77] Wishart, J.: The generalized product moment distribution in samples from a Normal multivariate population. Biometrika 20A, 32-52 (1928).

[78] Yau, H. T.: Relative entropy and the hydrodynamics of Ginzburg-Landau models, Lett. Math. Phys. 22 (1991) 63-80. MR1121850(93e:82035)

Institute of Mathematics, University of Munich, Theresienstr. 39, D-80333 Munich, GERMANY

E-mail address: lerdos@math.lmu.de

Department of Mathematics, Harvard University, Cambridge, Massachusetts 02138

E-mail address: htyau@math.harvard.edu 